\title{
Effects of phenylethanol glycosides from Orobanche cernua Loefling on UVB-Induced skin photodamage: a comparative study
}

\author{
Wei Gao ${ }^{1}$. Shengdao Zheng ${ }^{2}$. Eunson $\mathrm{Hwang}^{2} \cdot{\text { Tae-hoo } \mathrm{Yi}^{2} \cdot \text { Yu-shuai Wang }}^{1}$
}

Received: 31 January 2021 / Accepted: 24 March 2021 / Published online: 28 April 2021

(c) The Author(s), under exclusive licence to European Photochemistry Association, European Society for Photobiology 2021

\begin{abstract}
Previous study has found that Orobanche cernua Loefling $(\mathrm{OC})$ and its main ingredient, acteoside, possess excellently antiphoto-aging effect. In addition to acteoside, crenatoside, isoacteoside and 2'-acetylacteoside were also identified as the main phenylethanol glycosides (PhGs) in OC. To screen optimum effective substance and further clarify the photoprotective ingredients of OC, the effects of four major PhGs in OC were compared using UVB-irradiated HaCaT cells. Results indicated that acteoside, isoacteoside and 2'-acetylacteoside effectively decreased UVB-induced MMP-1 expression and stimulated type I procollagen synthesis through inhibition of MAPK/AP- 1 and activation of TGF- $\beta / S m a d$ pathway. Moreover, acteoside and 2 '-acetylacteoside significantly reduced UVB-induced ROS and TARC secretion, which is involved in the inhibition of NF- $\kappa \beta /$ I $\kappa \alpha$ and stimulation of Nrf2 antioxidant defense system. However, crenatoside did not show any effect on the regulation of signal cascades mentioned above. Together, our results suggested that 2 -acetylacteoside and isoacteoside also served as efficient agents against UV radiation-induced skin damage. Among them, acteoside and 2'-acetylacteoside showed a higher efficiency than that of isoacteoside, which possessed great potential in treating skin photo-damage.
\end{abstract}

Keywords Phenylethanol glycosides $\cdot$ Ultraviolet B $\cdot$ Photodamge $\cdot$ HaCaTs

\section{Introduction}

Photo-damage is a highly complex process in which multiple molecular mechanisms are involved. The key mechanism in which UV radiation caused skin damage lies in the excessive production of reactive oxygen species (ROS) [1]. It is reported that the UV-energized cellular chromophores can react with molecular oxygen, leading to ROS generation [2]. Actually, our skin is endowed with various antioxidant systems to deal with oxidative damage. The NF-E2-related

Wei Gao and Shengdao Zheng have contributed equally to this work.

Tae-hoo Yi

drhoo@khu.ac.kr

$\triangle$ Yu-shuai Wang

wangyushuai666@sina.com

1 Department of Pharmacy, Bengbu Medical College, 2600 Donghai Avenue, Bengbu 233030, China

2 Department of Oriental Medicinal Biotechnology, College of Life Sciences, Kyung Hee University, 1732, Deogyeong-daero, Giheung-gu, Yongin-si, Gyeonggi-do 17104, Republic of Korea nuclear factor 2 (Nrf2)/antioxidant-response element (ARE) pathway is one of the effective cellular defense systems, which is commonly seized by the Kelch-like ECH-associated protein 1 (Keap1) and localized in cytoplasm. Upon activation, $\mathrm{Nrf} 2$ is released from keap 1, trans-locates into the cell nucleus and binds to ARE, subsequently, activates antioxidants, such as HO-1 and NQO-1 expression [3]. When UV-generated ROS exceeds the capacity of cells to chemically eliminate ROS, damage will occur in skin. Long-time oxidative stress stimulates matrix metalloproteinase (MMPs) production, collagen degradation, finally leads to skin photoaging. Moreover, UV radiation causes cellular DNA damage, pigmentation, inflammation, immunomodulation, and even tumorigenesis [4].

The increase of MMPs caused by UV radiation was found to be induced by a series of protein kinase cascade $[5,6]$. Previous studies found that UVB-activated MAPK could trigger MMPs gene transcription by the up-regulation of activator protein 1 (AP-1) transcriptional factor [7]. Moreover, NF- $\kappa \beta$ was reported to be implicated in MMPs transcription [8]. NF- $\kappa \beta$ was a key transcription factor that regulates pro-inflammatory cytokines. It was commonly localized in cytoplasm by binding with an inhibitory protein 
known as inhibitory- $\kappa \beta(\mathrm{I}-\kappa \beta)$. UV radiation was found to activate NF- $\kappa \beta$ via stimulation of Iк $\beta$ degradation $[9,10]$. In addition to transcriptional regulation, the activated NF- $\kappa \beta$ also could accelerate MMPs expression by stimulation of inflammatory factors [11].

It is well known that UVB-irradiated skin could stimulate pro-inflammatory cytokines like IL- $1 \alpha, \mathrm{IL}-1 \beta$, TNF- $\alpha$. Thymus and activation-regulated chemokine (TARC) is produced in response to pro-inflammatory cytokines, especially TNF- $\alpha$ [12]. The TARC, also called CCL17, could attract CCR4-positive T cells known as Th2 subtype, which played a vital role in Th2-mediated skin inflammatory disease, such as atopic dermatitis (AD) [13]. Previous studies have reported that the narrowband UVB $(311 \mathrm{~nm})$ and UVA (320-400 nm) radiation could inhibit TNF- $\alpha$ and IFN- $\gamma$ induced TARC expression, which suggested that they have therapeutic effects on AD $[14,15]$. Unlike UVA and narrowband UVB, UVB irradiation was found to promote the development of AD-like skin lesions [16]. Recently, Jang et al. [17] found that UVB irradiation effectively increased thymic stromal lymphopoietin (TSLP) expression by activation MAPK pathway in HaCaTs. TSLP was the key factor to induce TARC expression [18], which suggested that MAPK might be involved in regulation of TARC expression.

Apart from promoting collagen degradation, UV radiation was also shown to suppress procollagen synthesis [19]. Transforming growth factor- $\beta$ (TGF- $\beta$ ) is an important regulator of procollagen synthesis [20]. It is reported that TGF- $\beta$ protein can initiate its cellular actions by combing with its cell-surface receptor, and then phosphorylate Smad2/3 transcription factor. The activated Smad2 and Smad3 associates with Smad4, in turn, transduces signal to nucleus and promote TGF- $\beta$-responsive genes transcription, such as type I procollagen [21]. Smad7 is a negative regulator that interfere $\mathrm{Smad} 2 / 3$ activation. UVB radiation was found to impair TGF- $\beta /$ Smad pathway through inhibition of $\mathrm{Smad} 2 / 3$ activation and up-regulation of Smad7 level [22].

The main ingredients of Orobanche cernua (OC) were identified as phenylethanoid glycosides (PhGs). HPLC showed that OC mainly contained crenatoside, acteoside, isoacteoside and 2'-acetylacteoside [23]. Previous study has found that $\mathrm{OC}$ and its main ingredient, acteoside, possess significant anti-photo-aging effects [24]. However, the effects of other compounds against UVB-induced skin photo-damage has not been studied. This study was performed to compare the photoprotective effects of main PhGs (crenatoside, acteoside, isoacteoside and 2'-acetylacteoside) from OC on UVB-induced photo-damage in HaCaTs, together with their underlying mechanism of action. The results indicated that acteoside, isoacteoside and 2'-acetylacteoside were more effective than crenatoside in regulating UV-induced skin photo-damage. They could effectively decrease UVB-induced MMP-1 expression via inhibition of MAPK/AP-1 pathway, and promoting procollagen type I synthesis through activation of TGF- $\beta /$ Smad. It was worth mentioning that acteoside and 2'-acetylacteoside could significantly inhibit UVB-induced ROS and TARC production, the internal mechanism was involved in the activation of Nrf2 antioxidant system and inhibition of NF- $\kappa \beta / \mathrm{I} \kappa \beta \alpha$ and MAPK pathway. Based on the present results, acteoside and 2 -acetylacteoside have shown more widely effects in preventing UVB-induced photo-damage, which served as good candidates in cosmetics and functional foods industry, as well as drugs developing.

\section{Materials and Methods}

\subsection{Chemicals and reagents}

The chemical reagents were provided by J.T. Baker (Avantor Performance Materials, Inc, Center Vally, PA, USA). An analytical-grade ethanol was purchased from Samchun Chemicals (Seoul, Korea). Dulbecco's modified Eagle's medium (DMEM), fetal bovine serum (FBS) and penicillin-streptomycin were obtained from Gibco BRL (Grand Island, NY, USA). The standard compounds of crenatoside, acteoside, isoacteoside and 2'-acetylacteoside were provided by our previous laboratory. The MMP-1 and TARC ELISA kits were purchased from R\&D Systems (R\&D Systems, Inc., Minneapolis, MN, USA). The Antibodies of

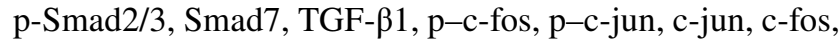
and $\beta$-actin were obtained from Santa Cruz Biotechnology (Dallas, TX, USA). The antibodies against p-ERK, p-JNK, p-p38, ERK, JNK, p38, Nrf2, HO-1, NQO-1, Histone, and SP600125 (JNK inhibitor), PD98059 (ERK inhibitor) were bought from Cell Signaling Technology (Danvers, MA, USA).

\subsection{Preparation of $O C$ extracts}

The OCs were kindly provided by Institute of Special Wild Economic Animals and Plants, Chinese Academy of Agricultural Sciences, which was identified by Prof. Wei Hou. The dried slices of OC $(20 \mathrm{~g})$ were powdered and extracted by $1 \mathrm{~L} 70 \%$ ethanol for $48 \mathrm{~h}$. The extracts were filtered and then evaporated under vacuum at $40{ }^{\circ} \mathrm{C}$ using a rotary evaporator. The concentrated extracts were finally lyophilized by a freeze dryer (FDCF-12012; Operon Co., Gimpo-si, Korea) for $48 \mathrm{~h}$.

\subsection{HPLC analysis}

High-performance liquid chromatography (HPLC) was performed on a Dionex Chromelon TM chromatography data system with P580 and UVD100 detectors (Thermo 
Fisher Scientific Inc., Waltham, MA USA). Chromatographic separation was performed on a Waters Sunfire C18 column $(250 \times 4.6 \mathrm{~mm}, 5-\mu \mathrm{m}$ particle size $)$. The mobile phase consisted of solvent A (Water with $0.1 \%$ formic acid, $\nu / \nu)$ and solvent $\mathrm{B}(\mathrm{MeOH})$ was eluted with the condition as follows:0-4 $\min , 30 \% \mathrm{~B}$; $4-8 \mathrm{~min} 30-38 \% \mathrm{~B} ; 8-22 \mathrm{~min}$, $38-40 \% \mathrm{~B} ; 22-24 \mathrm{~min}, 40-90 \% \mathrm{~B} ; 24-28 \mathrm{~min}, 90 \% \mathrm{~B}$ 28-29 min, 90-30\% B; 29-35 min, 30\% B. The injection volume was $10 \mu \mathrm{L}$ and the flow rate was $1 \mathrm{~mL} / \mathrm{min}$. The detection wavelength was set as $330 \mathrm{~nm}$, and the column temperature was $30^{\circ} \mathrm{C}$.

\subsection{Standard preparation and calibration curve}

The reference standards (crenatoside, acteoside, isoacteoside and 2'-acetylacteoside) were purified by our laboratory, and its purity was over $98 \%$ as determined by HPLC and ${ }^{1} \mathrm{H}$-NMR [23]. The chemical structural formulas of the four compounds were showed in Fig. 1. Standard stock solution of $1 \mathrm{mg} / \mathrm{mL}$ was prepared by dissolving $25 \mathrm{mg}$ standard substrate with $80 \%$ ethanol, and dilute to volume in a $25-\mathrm{mL}$ volumetric flask. Then, the stock was diluted to 500, 200, $100,50 \mathrm{ug} / \mathrm{ml}$, respectively. These four standard solutions together with the stock solution were injected to HPLC to generate a five point calibration curve. All standard curves possessed good linearity in that $R^{2}>0.999$. The areas of four compounds in $\mathrm{OC}$ were within the linear range of the standard curve. Relative standard deviations of the four compounds for three injections of the OC extract were less than $2.0 \%$.

\subsection{Dipheny1-1-picrylhdrazyl (DPPH) scavenging activity}

The antioxidant activities of crenatoside, acteoside, isoacteoside and 2'-acetylacteoside were determined by DPPH assay. $40 \mu \mathrm{L}$ of different concentration samples was put into a 96-well plate and then $160 \mu \mathrm{L}$ of DPPH $(0.2 \mathrm{mM})$ in methanol was added in each well. After incubation in the dark environment for $30 \mathrm{~min}$ at $37{ }^{\circ} \mathrm{C}$, the absorbance was measured at $520 \mathrm{~nm}$ by a microplate reader (Molecular Devices E09090; San Francisco, CA, USA). Arbutin as a positive control was introduced.

\subsection{Cell culture, UVB irradiation and sample treatment}

HaCaT cells (Sciencell, Carlsbad, CA, USA) were grown in DMEM containing 10\% heat-inactivated FBS and 1\% penicillin-streptomycin in a humidified atmosphere with $5 \% \mathrm{CO}_{2}$ and $95 \%$ air at $37{ }^{\circ} \mathrm{C}$. When the cell fusion reached $80 \%$, the media were removed and washed with PBS twice. Then, a thin layer of PBS was added into cell culture plate and cells were exposed to UVB radiation at $125 \mathrm{~mJ} / \mathrm{cm}^{2}$ by
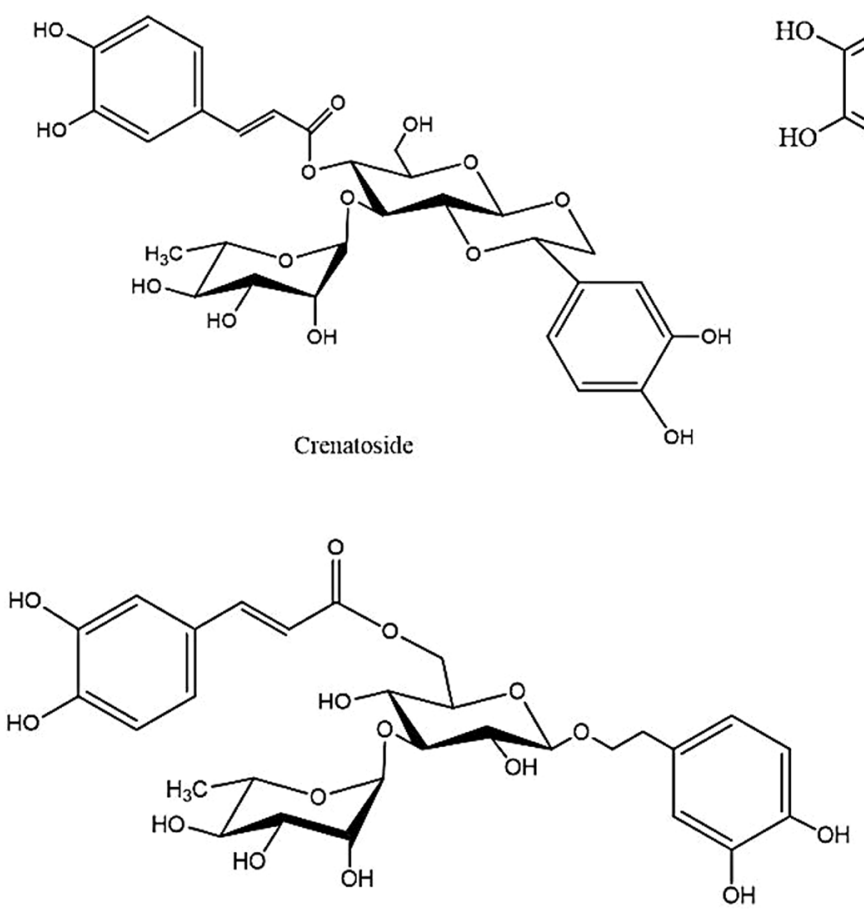

Isoacteoside

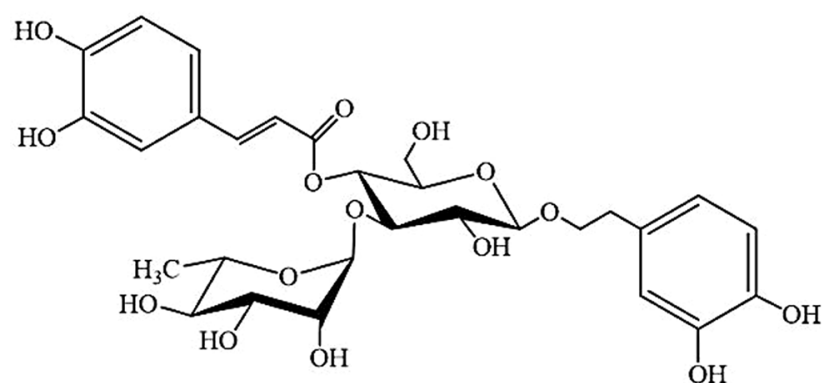

Acteoside

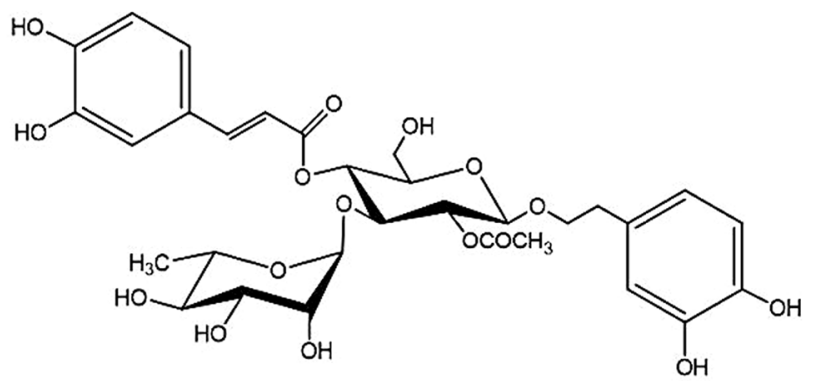

2'-acetylacteoside

Fig. 1 The chemical structural formula of crenatoside, acteoside, isoacteoside and $2^{\prime}$-acetylacteoside 
a UVB radiation machine (Bio-Link BLX-312; Vilber Lourmat GmbH, Marne-la-Vallee Fance). After irradiation, PBS was removed and treated with or without samples in serumfree medium conditions immediately. The normal group was kept in the same culture conditions without samples and UVB irradiation. To inhibit the function of JNK, HaCaTs cells were pre-treated with MAPK inhibitors $(20 \mu \mathrm{M})$ for $2 \mathrm{~h}$. In this study, the concentrations of all compounds were calculated as $\mu \mathrm{M}$.

\subsection{Cell viability}

The effects of crenatoside, acteoside, isoacteoside and 2 '-acetylacteoside on the viabilities of $\mathrm{HaCaTs}$ cells were assessed by MTT assay. MTT is a colorimetric assay for evaluating cell viability. The cell survival rate can be obtained through measuring the absorbance of colored solution at a certain wavelength. After $48 \mathrm{~h}$ samples' treatment, the supernatants of each well were removed entirely and a total of $100 \mu \mathrm{L}$ MTT solution $(0.1 \mathrm{mg} / \mathrm{mL})$ was added. After $4 \mathrm{~h}$ of incubation, the supernatants were aspirated and $150 \mu \mathrm{L}$ dimethyl sulfoxides was added. The absorbance was measured at $570 \mathrm{~nm}$ by a microplate reader (Molecular Devices, USA).

\subsection{Measurement of ROS production}

The intracellular ROS was measured by $2^{\prime} 7^{\prime}$-dichlorofluorescein diacetate (DCFH-DA). After $24 \mathrm{~h}$ samples treatment, the cells were incubated with $30 \mu \mathrm{M}$ of DCFH-DA (SigmaAldrich) at $37^{\circ} \mathrm{C}$ for $30 \mathrm{~min}$. Then, cells were rinsed with PBS three times and read by a multi-mode microplate reader as per the manufacturer's instruction (Molecular Devices Filter Max F5; Sunnyvale, CA, USA).

\subsection{Measurement of MMP-1 and TARC production}

After $72 \mathrm{~h}$ samples treatment, the supernatants of cell culture were harvested. The secretions of MMP-1 and TARC were detected using commercially available ELISA kits (R\&D Systems, Inc., Minneapolis, MN, USA) according to the manufacturer's instruction. Each experiment was repeated three times.

\subsection{Reverse transcription-polymerase chain reaction (RT-PCR)}

After $24 \mathrm{~h}$ samples treatment, the total RNA was isolated from $\mathrm{HaCaT}$ cells following the manufacturer's instructions by TRIZOL reagent (Invitrogen Life Technologies, Carlsbad, CA). The RNA ( $3 \mu \mathrm{g})$ was reverse-transcribed with 200 units of reverse transcriptase and $0.5 \mu \mathrm{g} / \mu \mathrm{L}$ oligo-(dT) 15 primer (Bioneer Co., Daejeon, Korea). This reaction was performed at $70{ }^{\circ} \mathrm{C}$ for $5 \mathrm{~min}, 42{ }^{\circ} \mathrm{C}$ for $60 \mathrm{~min}$, and then terminated at $94{ }^{\circ} \mathrm{C}$ for $5 \mathrm{~min}$. The PCR amplification performed with PCR premix (Bioneer Co., Daejeon, Korea), and the primers used this reaction are described as follows: GAPDH forward primer 5'-ACC ACA GTC CAT GCC ATC AC-3', reverse primer 5'-CCA CCA CCC TGT TGC TGT AG-3'; MMP-1 forward primer 5'-TGC GCA CAA ATC CCT TCT AC-3', reverse primer 5'-TTC AAG CCC ATTTGG CAG TT-3'; Type I procollagen forward primer 5'- CTC GAG GTG GAC ACC CT-3', reverse primer 5'-CAG CTG GAT GGC CAC ATC GG - $3^{\prime}$

\subsection{Western blot analysis}

The HaCaT cells were harvested after samples treatment. Total proteins were extracted and analyzed by western blot as previously methods [25]. Densitometric measurements of bands were analyzed by ImageMaster TM 17 2D Ekite software (Amersham Pharmacia Biotech, Piscataway, NJ, USA). Each experiment was repeated at least three times.

\subsection{Statistical analysis}

All experiments were performed independently three times. Differences in data among the groups were analyzed by one-way analysis of variance ANOVA, and all values were expressed as mean \pm S.D. $p<0.05, p<0.01$ and $p<0.001$ were considered statistically significant for $t$ test.

\section{Results}

\subsection{Analysis of main components from OC}

The major compounds isolated from OC were identified as crenatoside, acteoside, isoacteoside and 2'-acetylacteoside (Fig. 2). The contents of these four compounds were confirmed by comparing the retention time and the spectrum with the standard offered by the Institute of Special Animal and Plant Sciences of CAAS. The purity of these compounds is over $98 \%$ as detected by area normalization method based on the HPLC chromatogram. The contents of acteoside, isoacteoside, crenatoside, and 2'-acetylacteoside were $62.85,63.10,27.72$ and $14.90 \mu \mathrm{g} / \mathrm{g}$, respectively. Total phenylethanoid glycosides were calculated as $168.57 \mu \mathrm{g} / \mathrm{g}$.

\subsection{DPPH radical scavenging activity}

As shown in Fig. 3, crenatoside, acteoside, isoacteoside and 2 '-acetylacteoside does-dependently scavenged DPPH free radicals, they showed stronger scavenging abilities than arbutin. Crenatoside, acteoside, isoacteoside and 2'-acetylacteoside inhibited DPPH radicals with $\mathrm{IC}_{50}$ values of 

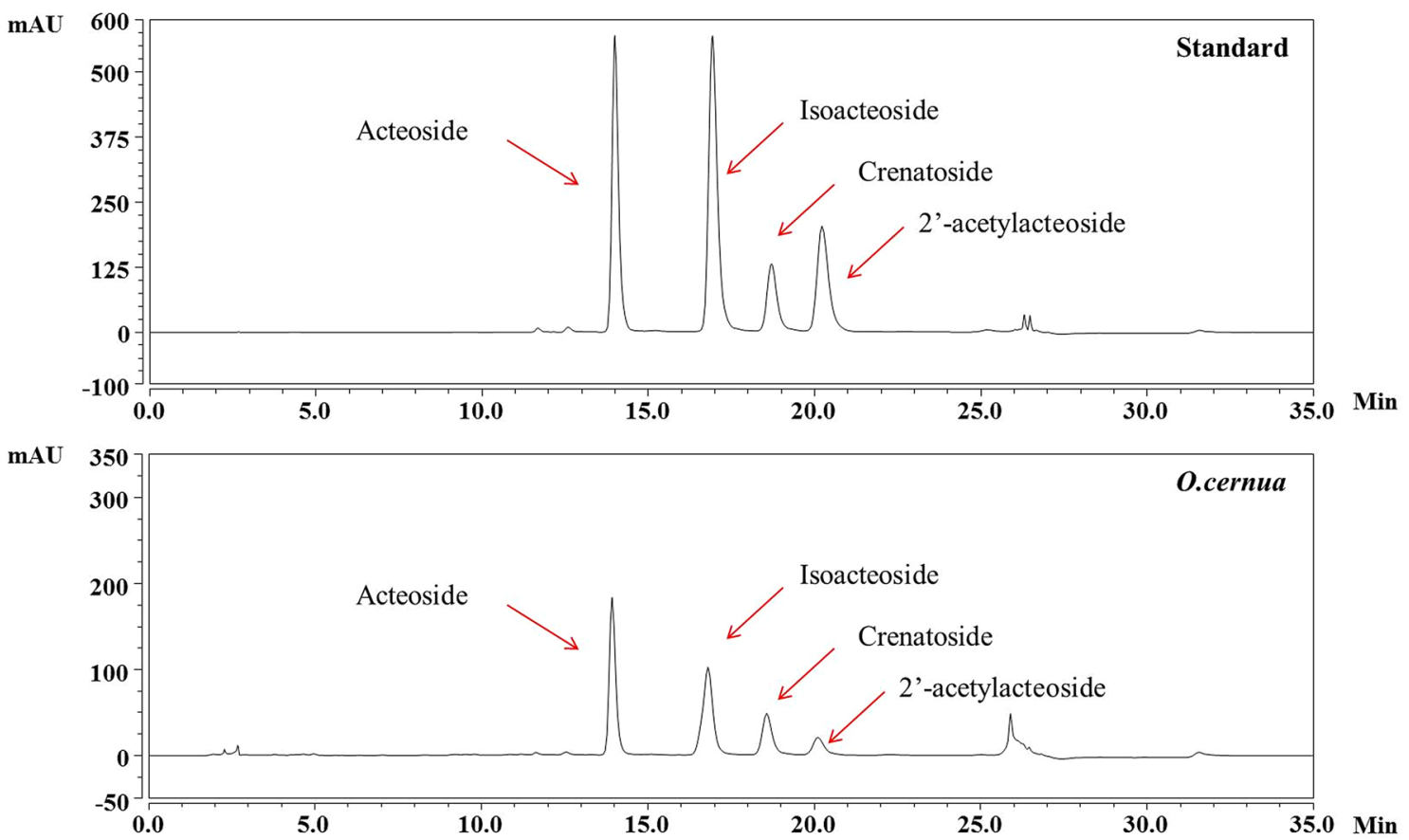

Fig. 2 HPLC profile of standard (crenatoside, acteoside, isoacteoside and 2'-acetylacteoside) and the extract derived from OC

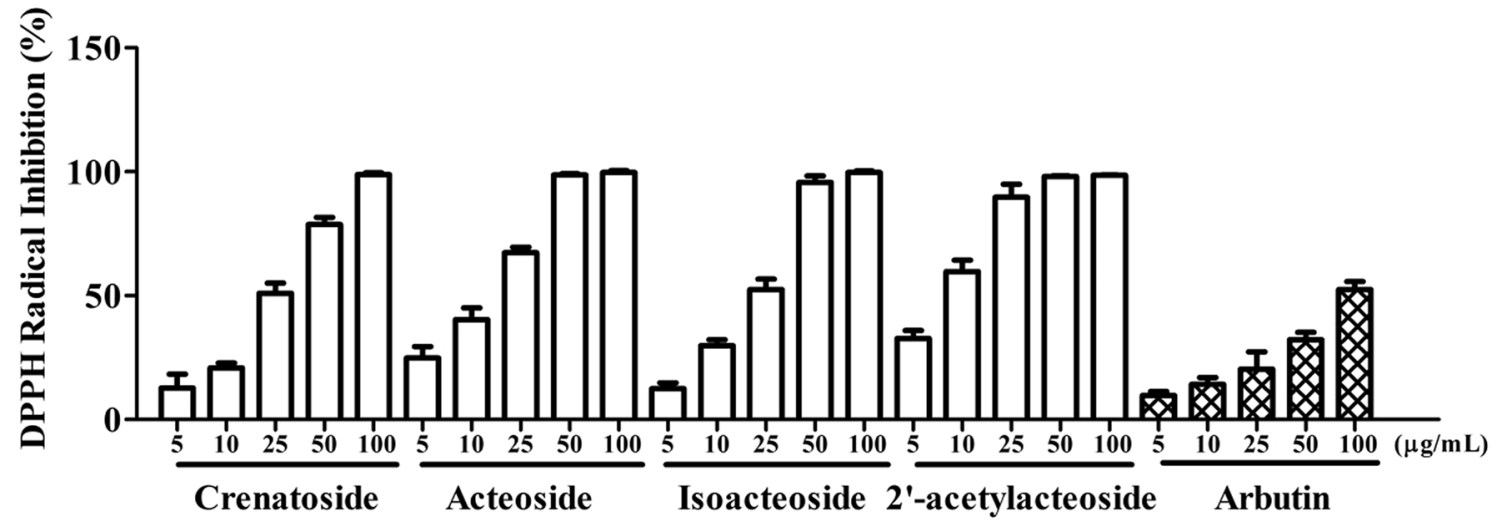

Fig. 3 Effects of crenatoside, acteoside, isoacteoside and 2'-acetylacteoside on DPPH radicals. The inhibitory activities were calculated as the $\%$ of normal value. The range of concentration was 5, 10,25, 50 and $100 \mu \mathrm{g} / \mathrm{mL}$. Abutin was used as a positive control. Values are means \pm SDs

$28.76 \mu \mathrm{g} / \mathrm{mL}, 17.59 \mu \mathrm{g} / \mathrm{mL}, 23.87 \mu \mathrm{g} / \mathrm{mL}$ and $9.30 \mu \mathrm{g} / \mathrm{mL}$, respectively.

\subsection{Cell viability}

MTT assay was conducted to analyze the effects of crenatoside, acteoside, isoacteoside and 2'-acetylacteoside on cell viabilities. As shown in Fig. 4, the HaCaT cell viability declined significantly after UVB radiation compared with the non-radiated cell; however, treatment of acteoside, isoacteoside and 2'-acetylacteoside reversed the decreased cellular activity in a dose-dependent manner. Acteoside, isoacteoside and 2 '-acetylacteoside with $10 \mu \mathrm{M}$ recovered the viability by $23.71 \%, 20.24 \%$ and $29.49 \%$, compared with UVB-irradiated cells.

\subsection{ROS production}

As shown in Fig. 5, treatment of cells with UVB radiation resulted in the increase of ROS levels, whereas acteoside and 2'-acetylacteoside $(10 \mu \mathrm{M})$ treatment markedly inhibited UVB-stimulated ROS production, the trend was reduced by $39.47 \%$ and $43.01 \%$, respectively, compared with UVBtreated group. 

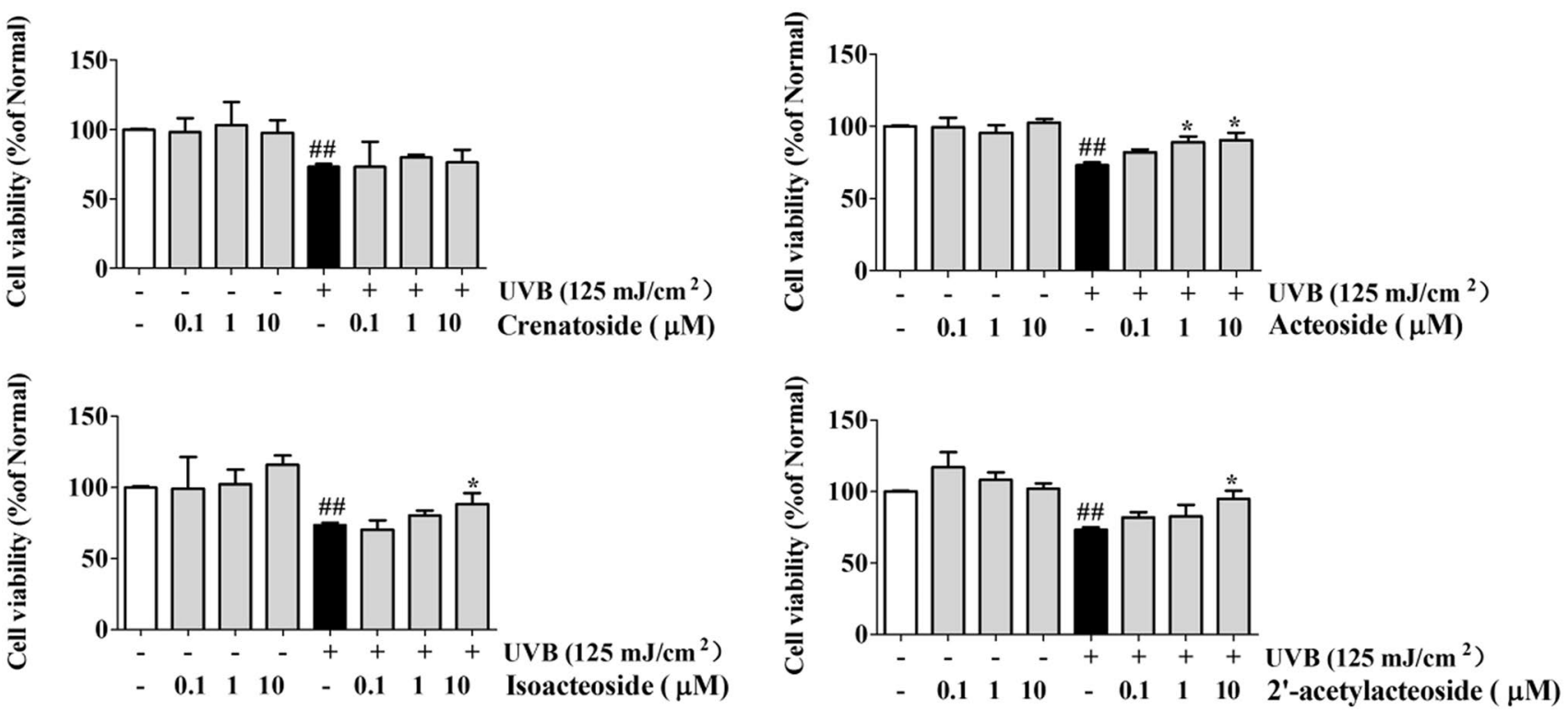

Fig. 4 Effects of crenatoside, acteoside, isoacteoside and 2'-acetylacteoside on cell viability. HaCaT cells were irradiated or non-irradiated with $125 \mathrm{~mJ} / \mathrm{cm}^{2}$ UVB. Then the cells were treated with different concentrations of samples for $48 \mathrm{~h}$. Cell viabilities were measured

by MTT assay. All data was shown as the mean \pm SD. \#\#=p<0.01, compared with non-irradiated group. $*=p<0.05$, compared with only UVB-irradiated group
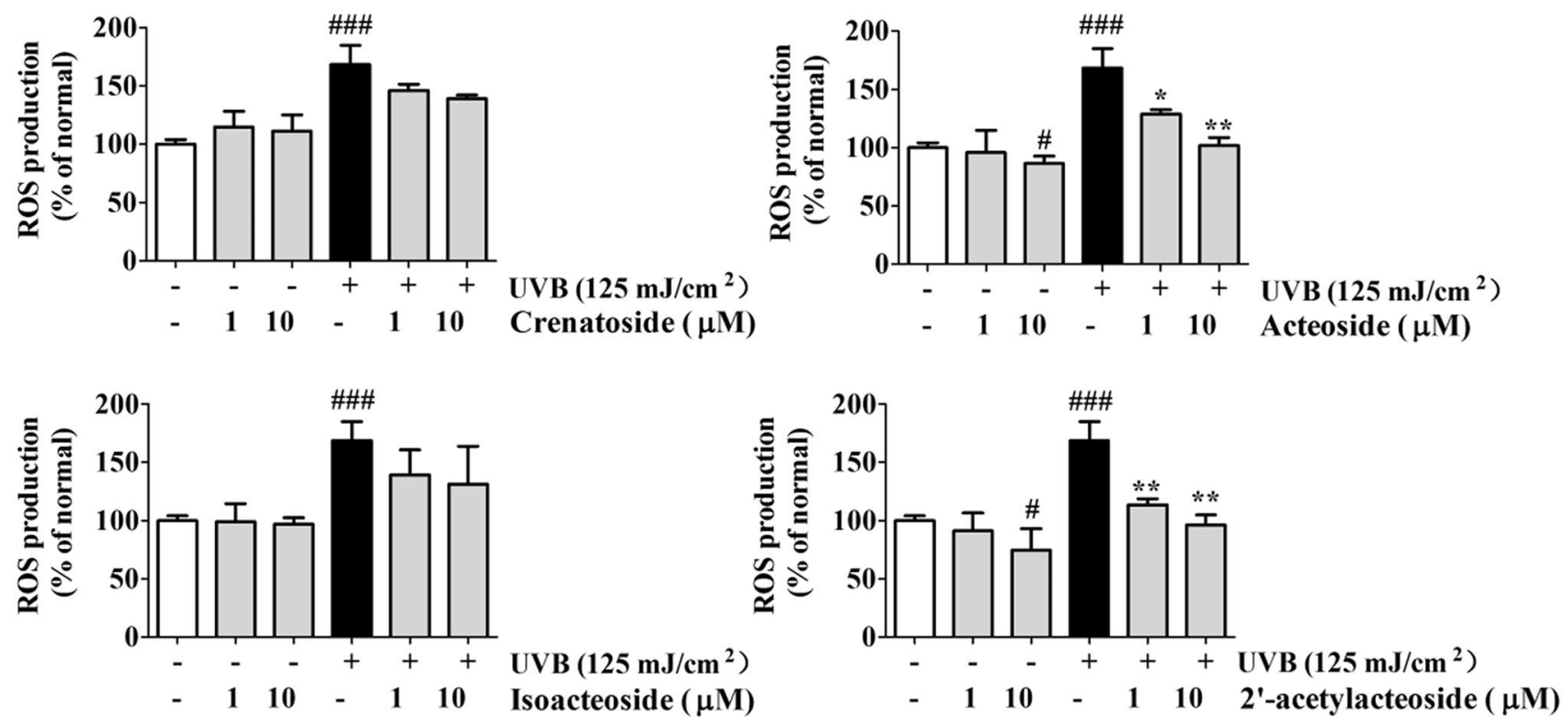

Fig. 5 Effect of crenatoside, acteoside, isoacteoside and 2'-acetylacteoside on ROS production. After UVB irradiation, the cells were treated with different concentrations of samples for $24 \mathrm{~h}$. Cellular ROS levels were measured by DCFH-DA assay. All data was shown

\subsection{MMP-1 production detected by ELISA kit}

The effects of crenatoside, acteoside, isoacteoside and 2 -acetylacteoside on MMP-1 expression were investigated by ELISA kit. As shown in Fig. 6, UVB-irradiated cells

as the mean \pm SD. $\#=p<0.05, \# \#=p<0.001$, compared with nonirradiated group. $*=\mathrm{p}<0.05, * *=\mathrm{p}<0.01$, compared with only UVB-irradiated group

caused a significant increase in MMP-1 secretion. However, treatment with acteoside, isoacteoside and 2'-acetylacteoside significantly prevented the cells from UVB-induced MMP-1 production. It was indicated that $10 \mu \mathrm{M}$ acteoside, isoacteoside and 2'-acetylacteoside could reduce UVB-induced 

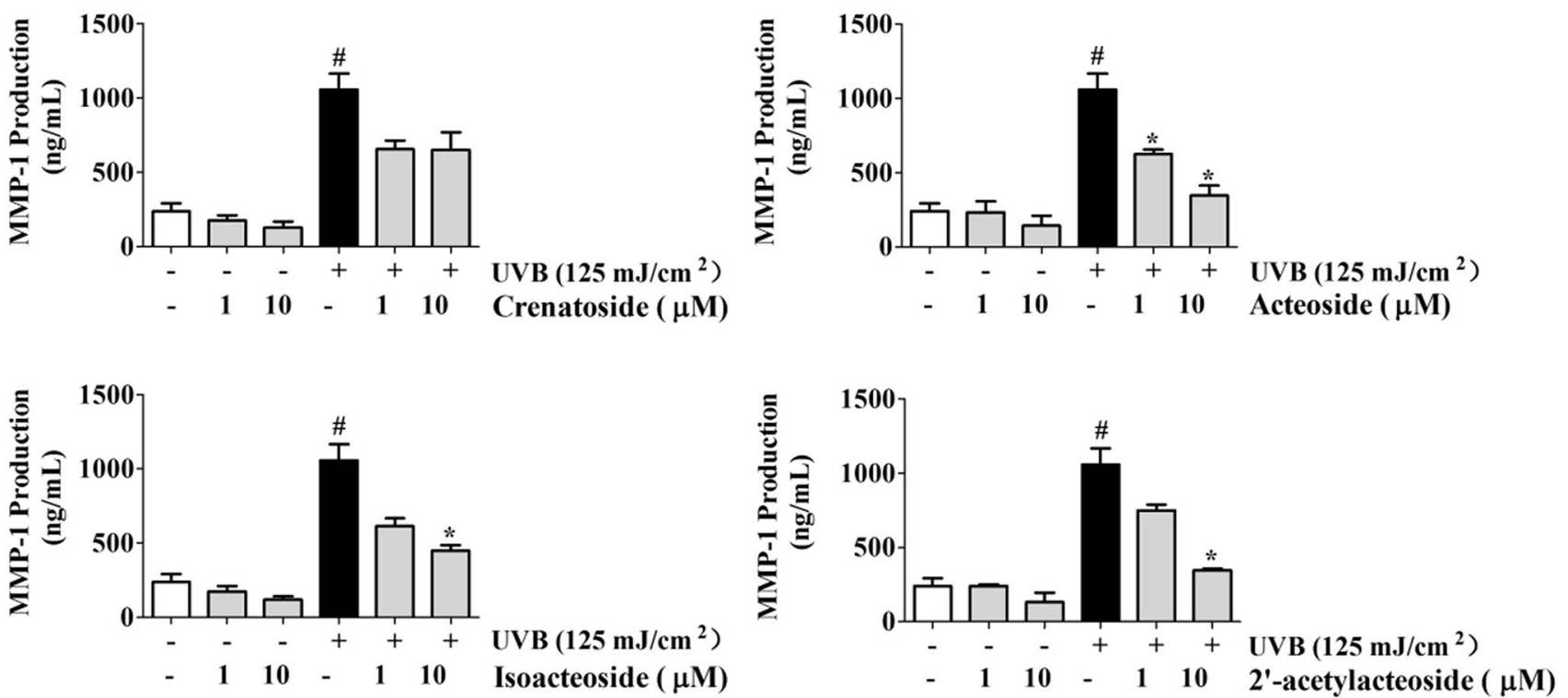

Fig. 6 Effects of crenatoside, acteoside, isoacteoside and 2'-acetylacteoside on MMP-1 production. After UVB irradiation, the cells were treated with different concentrations of samples for $24 \mathrm{~h}$.

MMP-1 secretion was measured by ELISA kit. All data was shown as the mean \pm SD. $\#=p<0.05$, compared with non-irradiated group. $*=p<0.05$, compared with only UVB-irradiated group

MMP- 1 expression by $67.29 \%, 57.32 \%$ and $67.20 \%$, respectively, compared with UVB group.

\subsection{TARC production detected by ELISA kit}

To determine whether PhGs inhibited UVB-induced TARC production, the supernatants were evaluated by ELISA kit.

As shown in Fig. 7, the level of TARC was increased greatly after UVB irradiation. Treatment of acteoside, and 2'-acetylacteoside decreased TARC expression in a dose-dependent manner. The results indicated that $10 \mu \mathrm{M}$ acteoside and 2 '-acetylacteoside could reduce UVB-induced TARC expression by $45.07 \%$ and $51.59 \%$, respectively, compared with UVB group.
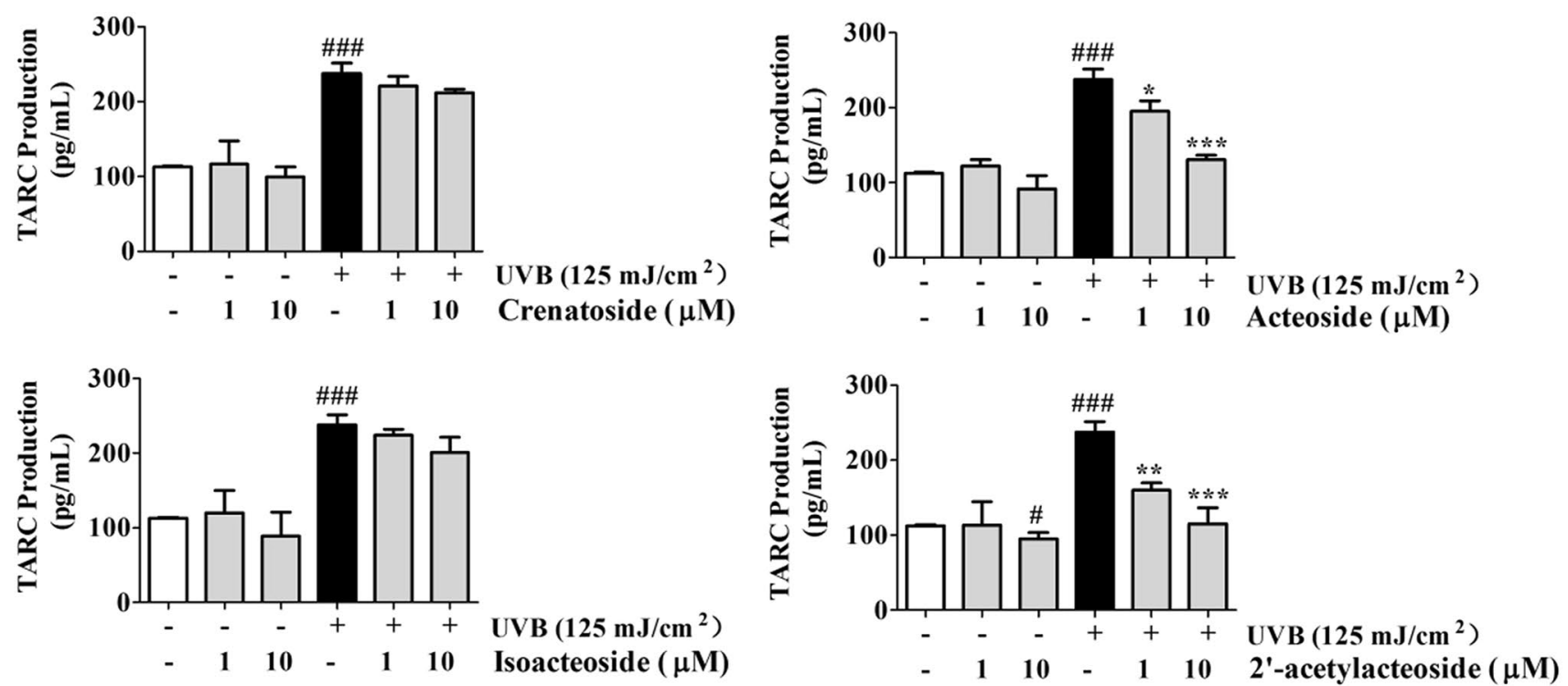

Fig. 7 Effects of crenatoside, acteoside, isoacteoside and 2'-acetylacteoside on TARC production. After UVB irradiation, the cells were treated with different concentrations of samples for $24 \mathrm{~h}$. TARC secretion was measured by ELISA kit. All data was shown as the

mean \pm SD. $\#=p<0.05, \# \#=p<0.001$, compared with non-irradiated group. $*=\mathrm{p}<0.05, * *=\mathrm{p}<0.01, * * *=\mathrm{p}<0.001$, compared with only UVB-irradiated group 


\subsection{MMP-1 and Type I procollagen mRNA expression analyzed by RT-PCR}

The effects of PhGs on the mRNA levels of MMP-1 and Type I procollagen in UVB-irradiated $\mathrm{HaCaTs}$ were assessed by RT-PCR. As shown in Fig. 8, the mRNA level of MMP-1 was observably enhanced when $\mathrm{HaCaTs}$ were treated with $125 \mathrm{~mJ} / \mathrm{cm}^{2}$ UVB. However, this increase in MMP-1 was significantly attenuated by treatment with acteoside, isoacteoside and 2'-acetylacteoside, which could inhibit MMP-1 expression by $24.14 \%, 31.28 \%$ and $36.54 \%$, respectively, compared with UVB-irradiated groups.

Conversely, the mRNA level of type I procollagen was decreased after UVB irradiation. The results demonstrated that post-treatment with acteoside, isoacteoside, 2 '-acetylacteoside could reverse UVB-induced decrease of type I procollagen mRNA. Specifically, acteoside $(10 \mu \mathrm{M})$ could effectively promote type I procollagen levels by $34.98 \%$, compared with the UVB group.

\subsection{Effects on MAPK pathway}

The effects of PhGs on MAPK signaling pathway were investigated with western blotting. The results revealed that the phosphorylation levels of ERK, JNK and p-p38 were significantly increased after UV irradiation compared with that of the normal group (Fig. 9). Acteoside, isoacteoside and 2 '-acetylacteoside effectively reversed the up-expression of p-ERK, p-JNK, and p-p38 induced by UVB radiation. However, cells treated with crenatoside attenuated UVBinduced phosphorylation of $\mathrm{p} 38$ and JNK, but did not affect the phosphorylation of ERK.

\subsection{Effects on AP-1 pathway}

To examine whether acteoside, isoacteoside and 2'-acetylacteosid inhibited MMP-1 expression by blocking AP-1 activity, the effects of PhGs on the expression of the composition of AP-1 were detected by western blotting. The results found that post-treatment with acteoside, isoacteoside and 2'-acetylacteoside effectively attenuated UVB-induced $\mathrm{p}-\mathrm{c}$-jun expression (Fig. 10). The p-c-fos expression was inhibited by acteoside and 2'-acetylacteoside treatment. However, crenatoside did not show any effect on AP-1 activity, compared with UVB group. Accordingly, these results suggested that acteoside and 2'-acetylacteoside showed effective inhibitory effect on UVB-induced AP-1 activation.
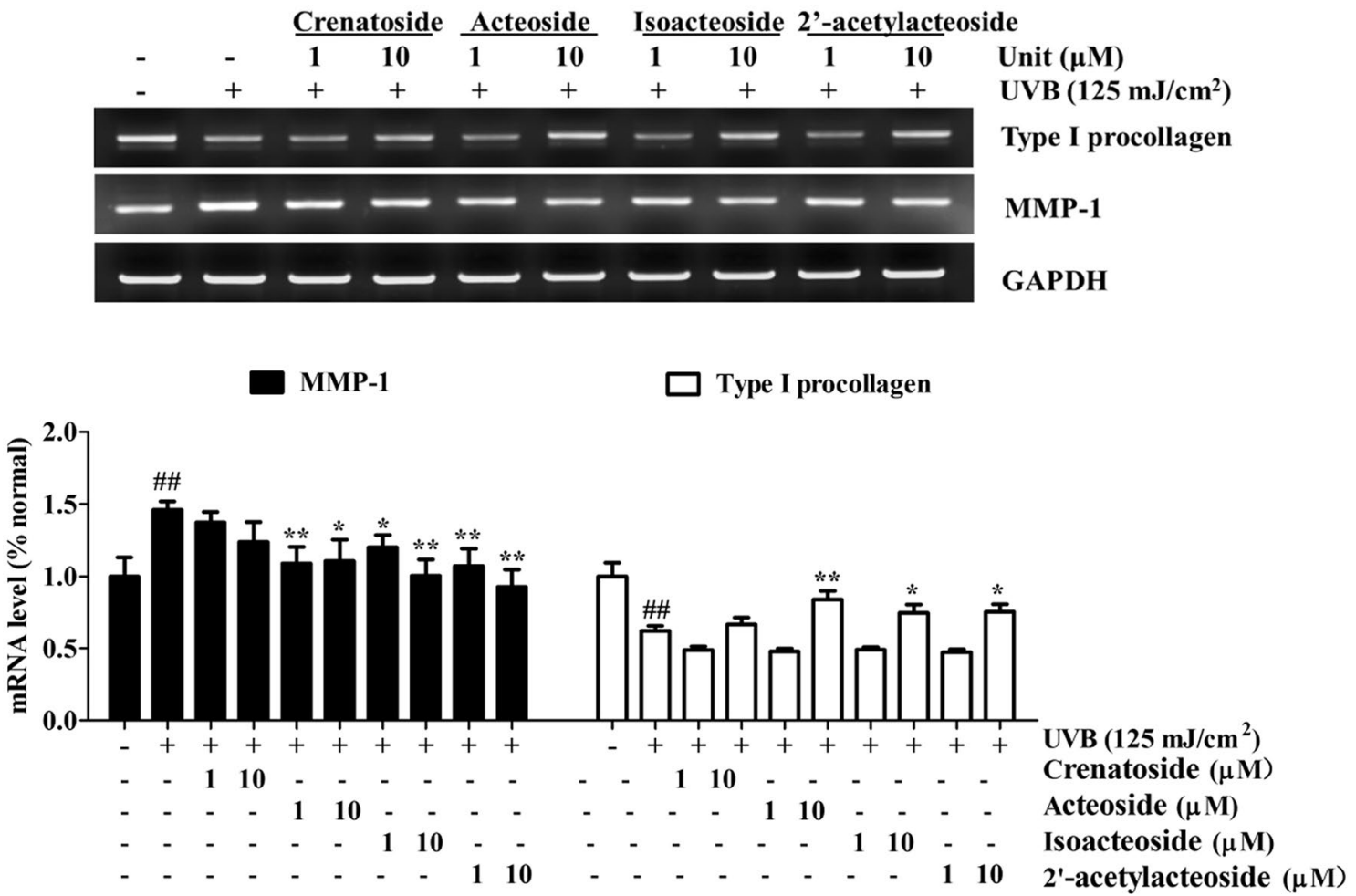

Fig. 8 Effects of crenatoside, acteoside, isoacteoside and 2'-acetylacteoside on MMP-1 and type I procollagen mRNA expression. After UVB irradiation, the cells were treated with different concentrations of samples for $24 \mathrm{~h}$. The mRNA levels of MMP-1 and type
I procollagen were measured by RT-PCR. All data was shown as the mean $\pm \mathrm{SD}$. $\# \#=p<0.01$, compared with non-irradiated group. $*=p<0.05, * *=p<0.01$, compared with only UVB-irradiated group 


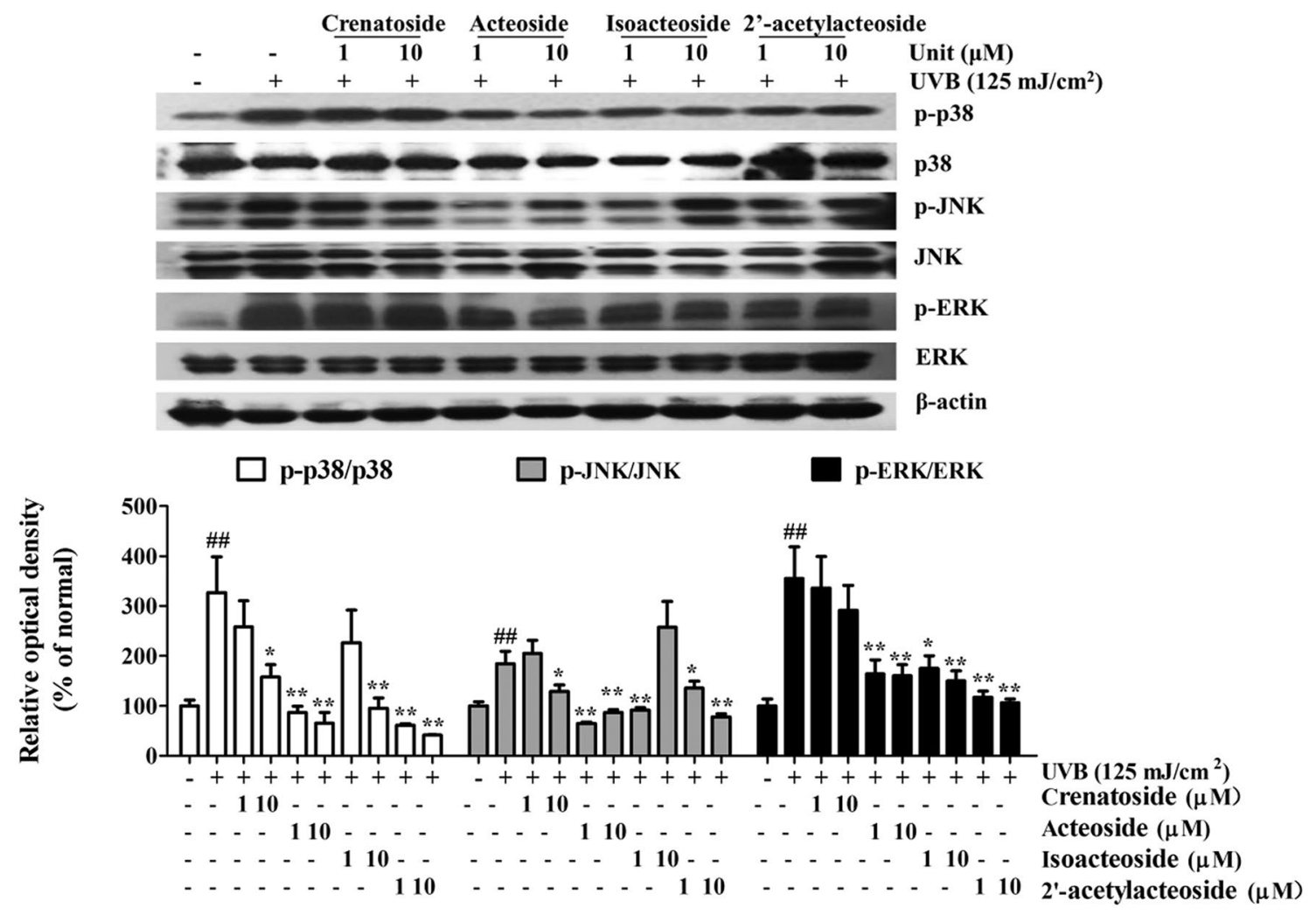

Fig. 9 Effects of crenatoside, acteoside, isoacteoside and 2'-acetylacteoside on MAPK pathway. After UVB irradiation, the cells were treated with different concentrations of samples for $1 \mathrm{~h}$. The protein levels of p-38, p-JNK, p-ERK and their phosphorylation form were

\subsection{Effects on NF-k $\beta /$ Iкßa pathway}

To determine whether PhGs could inhibit UVB-induced MMP- 1 and TARC secretion by regulation of NF- $\kappa \beta / \mathrm{I} \kappa \beta \alpha$ pathway, the effects of PhGs on p-NF- $\kappa \beta$ and Iк $\beta \alpha$ expression were further investigated. The results indicated that acteoside and 2'-acetylacteoside effectively inhibited UVB-induced phosphorylation of NF- $\kappa \beta$. As shown in Fig. 11, acteoside and 2'-acetylacteoside decreased UVBinduced $\mathrm{p}-\mathrm{NF}-\kappa \beta$ expression by $50.24 \%$ and $78.99 \%$ at $10 \mu \mathrm{M}$, respectively. Crenatoside and isoacteoside did not show any effects on the expression of $\mathrm{p}-\mathrm{NF}-\kappa \beta$ in UVB-irradiated HaCaTs, compared with UVB group. Furthermore, the effects of crenatoside, acteoside, isoacteoside and 2 -acetylacteoside on Iк $\beta \alpha$ expression were also measured, which served as an inhibitory protein of NF- $\kappa \beta$. Interestingly, crenatoside promoted the up-regulation of I $\kappa \beta \alpha$ in UVB-irradiated HaCaTs, but the stimulating role was less effective than that of acteoside and 2 '-acetylacteoside. measured by western blot. All data was shown as the mean \pm SD . $\# \#=p<0.01$, compared with non-irradiated group. $*=p<0.05$, $* *=p<0.01$, compared with only UVB-irradiated group

\subsection{Effects on TGF- $\beta /$ Smad pathway}

To evaluate whether PhGs could stimulate procollagen type I synthesis through activation of TGF- $\beta /$ Smad pathway, western blotting was performed to investigate the effects of PhGs on TGF- $\beta /$ Smad signaling pathway. The results demonstrated that the levels of TGF- $\beta 1$ and $p-\operatorname{Smad} 2 / 3$ were decreased greatly after UVB radiation. However, these decreases could be recovered by acteoside and 2'-acetylacteoside treatment. In addition, crenatoside and isoacteoside also showed a repair effect on TGF- $\beta 1$ expression in that they increased the TGF- $\beta 1$ expression by $338.52 \%$ and $349.65 \%$ compared with the UVB group, respectively. Furthermore, the effects of PhGs on Smad7 expression were further investigated. It was worth mentioning that all compounds could inhibit UVB-induced Smad7 production effectively. Crenatoside, acteoside, isoacteoside and 2'-acetylacteoside at $10 \mu \mathrm{M}$ decreased Smad7 level by $64.63 \%$, $85.98 \%, 77.28 \%$ and $74.56 \%$, respectively, compared with UVB-treated group (Fig. 12). 

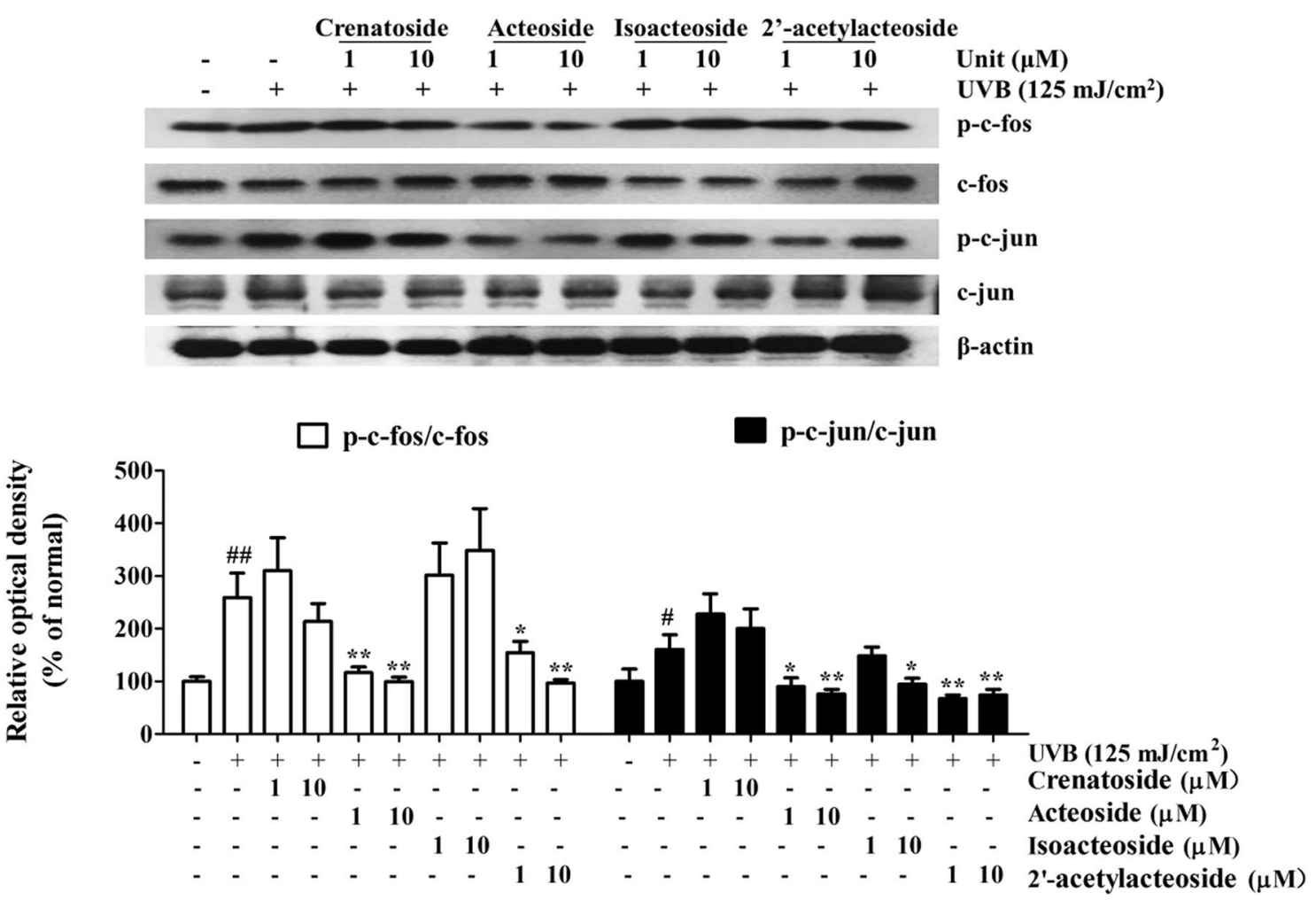

Fig. 10 Effects of crenatoside, acteoside, isoacteoside and 2'-acetylacteoside on AP-1 pathway. After UVB irradiation, the cells were treated with different concentrations of samples for $4 \mathrm{~h}$. The protein levels of c-fos, c-jun and their phosphorylation form were measured

\subsection{Effects on Nrf2 pathway}

Nrf2 is generally regarded as the primary defense system in our body, which is involved in regulation of multiple antioxidant enzymes, such as HO-1 and NQO-1 expression. We found that the expression of $\mathrm{Nrf} 2$ protein was significantly elevated when UVB-irradiated HaCaTs were treated with acteoside and 2'-acetylacteoside. Meanwhile, the levels of HO-1 and NQO-1 were also significantly enhanced as shown in Fig. 13. The result showed that $10 \mu \mathrm{M}$ of acteoside accelerated the expression of Nrf2, HO-1 and NQO-1 by $60.81 \%, 118.35 \%$ and $153.76 \%$, compared with UVB group, and $10 \mu \mathrm{M}$ of $2^{\prime}$-acetylacteoside showed increase rates of $46.72 \%, 111.05 \%$ and $174.51 \%$, respectively. Crenatoside and isoacteoside did not show any effects on the expression of Nrf2, HO-1 and NQO-1.

\subsection{MAP kinase inhibitors}

To further explore the role of MAPK pathway on MMP-1 and TARC regulation, the MMP- 1 and TARC expressions in cells exposed to UVB radiation in the presence and absence of JNK inhibitor (SP600125) and ERK inhibitor (PD98059) were measured. The result indicated that pre-treatment of by western blot. All data was shown as the mean $\pm \mathrm{SD}$. $\#=p<0.05$, $\# \#=p<0.01$, compared with non-irradiated group. $*=p<0.05$, $* *=p<0.01$, compared with only UVB-irradiated group

cells with PD98059 and SP600125 inhibitors $(20 \mu \mathrm{M})$ blocked the UVB-induced ERK and JNK phosphorylation (Fig. 14). As expected, treatment of HaCaTs with ERK and JNK inhibitors could effectively reduce UVB-induced MMP-1 secretion. As shown in Fig. 14, ERK and JNK inhibitors decreased MMP-1 expression by $49.01 \%$ and $67.21 \%$, respectively, compared with UVB group. On the other hand, JNK inhibitor also exhibited declined effects on TARC expression, which suggested that UVB-stimulated TARC expression was mediated by the activation of JNK. These results demonstrated that acteoside, isoacteoside and 2'-acetylacteoside could attenuate the expression of MMP-1 and TARC production by regulating the MAPK signaling pathway.

\section{Discussion}

UV radiation from the sun is commonly considered to be one of the most serious environmental threats that impact skin. Searching for natural and efficient agent against UV damage has attracted increasing attention. There has been considerable interest in applying botanical agents instead of synthetic chemical because of their safe and non-toxic advantages. 

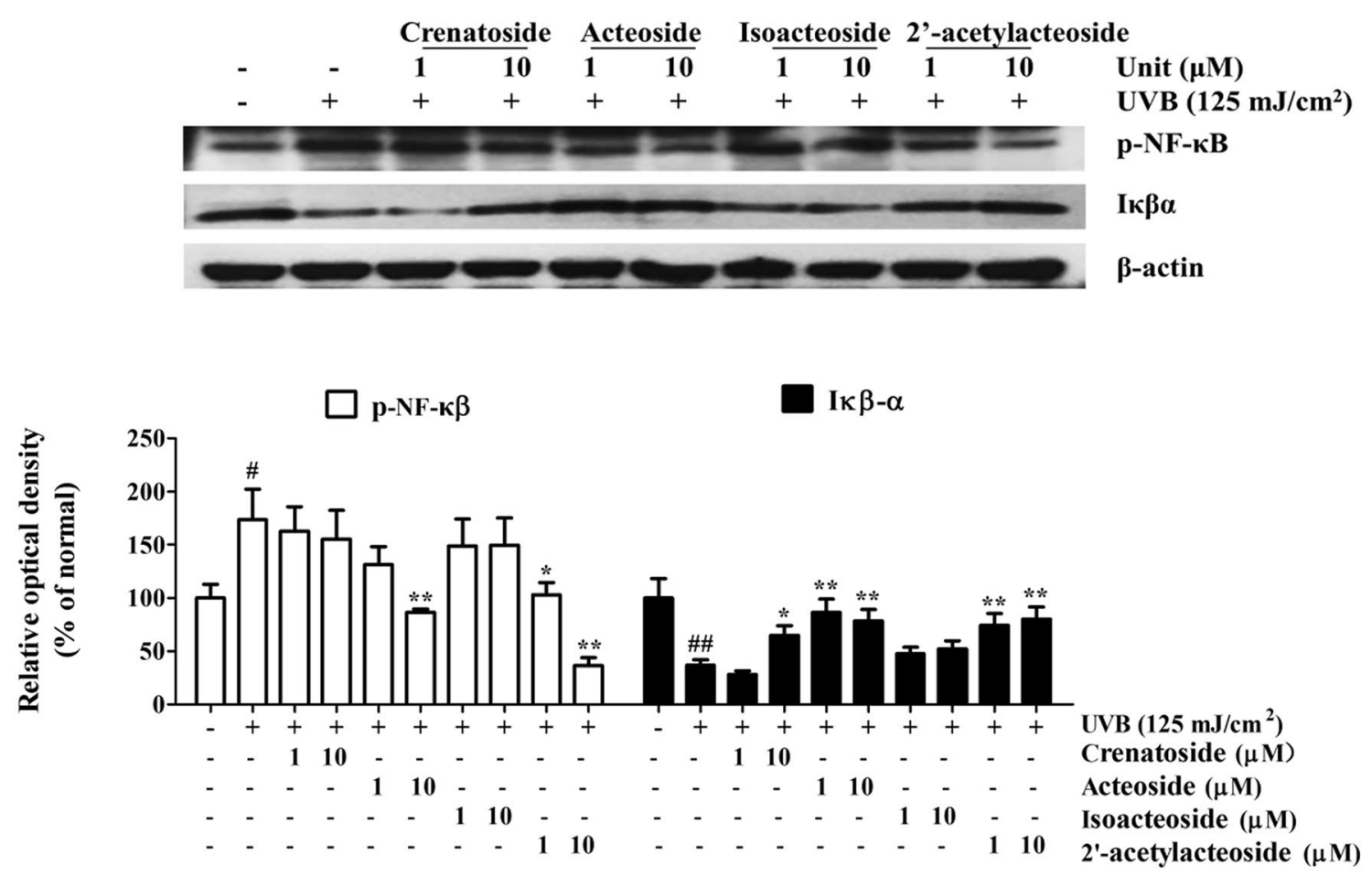

Fig. 11 Effects of crenatoside, acteoside, isoacteoside and 2'-acetylacteoside on Iк $\beta \alpha / \mathrm{NF}-\kappa \beta$ pathway. After UVB irradiation, cells were treated with different concentrations of samples for $3 \mathrm{~h}$. The levels of $\mathrm{p}-\mathrm{NF}-\kappa \beta$ and I $\kappa \beta \alpha$ were detected by western blotting. All data was shown as the mean $\pm \mathrm{SD}$. $\#=p<0.05, \# \#=p<0.01$, compared with non-irradiated group. $*=p<0.05, * *=p<0.01$, compared with only UVB-irradiated group

anti-inflammation and whitening activities. Chae et al. [30] have shown that isoacteoside effectively scavenged ROS, DPPH radical and prevented lipid peroxidation by increase of cellular antioxidant enzymes activities. Isoacteoside was also found to reduce pro-inflammatory cytokines, such as IL- $1 \beta$, IL-6, IL- 8 and TNF- $\alpha$ production, by inhibition of caspase-1, MAPK and NF- $\kappa \beta$ pathways in PMACI-stimulated HMC-1 cells [31]. Moreover, Shen et al. [32] have reported that crenatoside alleviated $\mathrm{CCl}_{4}$-induced hepatotoxicity by inhibition of the malondialdehyde, ROS production, as well as NF- $\kappa \beta$ transactivation. 2'-acetylacteoside was found to reduce the oxidative stress in the reperfusion-induced myocardial infarction by decrease of MDA levels and elevation of the activities of GSH-Px, SOD [33]. Overall, we deduced that the phenylethanoid glycosides possess multiple biological activities with certain application potentiality in skin photo-aging. To define the optimal agent against skin photo-damage, we compared the photo-protective effect of acteoside, crenatoside, isoacteoside and 2'-acetylacteoside on UVBirradiated HaCaTs, as well as the intrinsic mechanism.

It is well known that chronic exposure to UV radiation causes premature aging of skin. Previous studies suggested that the loss of collagen was the primary reason for photoaging. It is reported that UV could induce reduction of 

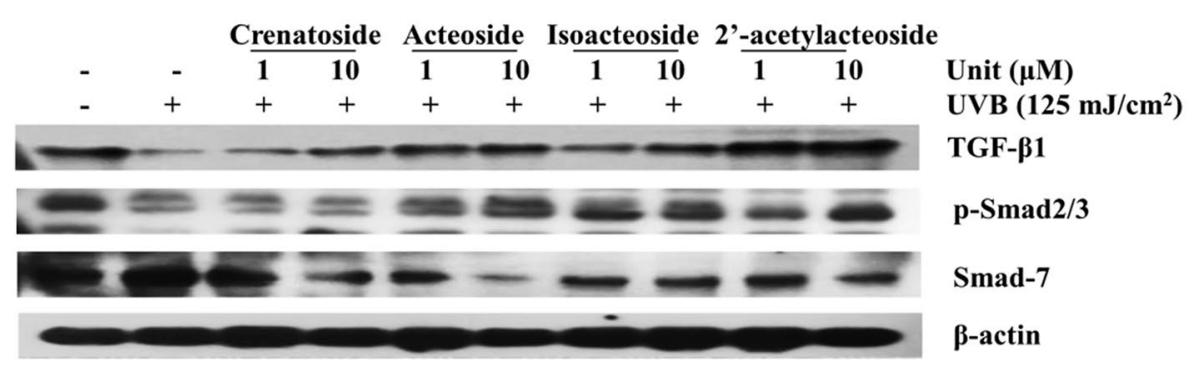

p-Smad2/3

Smad-7

$\beta$-actin

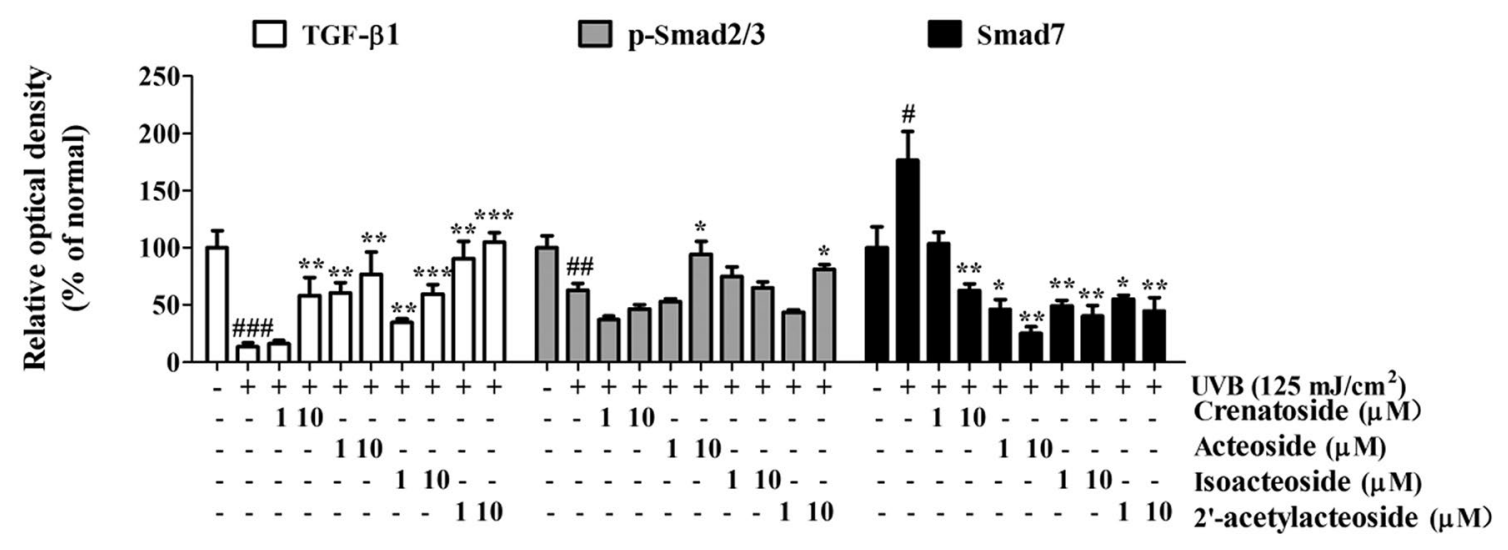

Fig. 12 Effects of crenatoside, acteoside, isoacteoside and 2'-acetylacteoside on TGF- $\beta /$ Smad pathway. After UVB irradiation, cells were treated with different concentrations of samples for $1.5 \mathrm{~h}$. The levels of TGF- $\beta 1$, p-Smad2/3 and Smad7 were detected by west-

collagen by two ways: stimulation of collagen degradation and suppression of procollagen synthesis.

A large number of reports indicated that the up-regulation of MMPs played a major role in extracellular matrix degradation [34]. MMP-1, as the critical member of MMPs family, is the major collagenolytic enzyme responsible for collagen destruction [35]. Here, UVB-induced MMP-1 expression was markedly inhibited by treatment of acteoside, isoacteoside and 2'-acetylacteoside. To further clarify the inhibitory mechanism on MMP-1, the effects on MAPK/ AP-1 and I $\kappa-\beta \alpha / N F-\kappa \beta$ signaling pathway were investigated on UVB-irradiated HaCaTs. The results found that acteoside, isoacteoside and 2'-acetylacteoside effectively alleviated UVB-induced MAPK/AP-1 activation (Figs. 9 and 10). Among them, acteoside and 2 'acetylacteoside could also regulate I $\kappa-\beta \alpha / \mathrm{NF}-\kappa \beta$ signaling pathway (Fig. 11). These results suggested that acteoside and 2'acetylacteoside were more efficient than other substance against UVB-induced photo-damage.

On the other hand, UV radiation has been reported to reduce procollagen synthesis by inhibiting TGF- $\beta / \mathrm{Smad}$ signaling pathway [36]. Whether PhGs have a repair effect on UVB-induced downregulation of procollagen synthesis was further investigated. The results indicated that acteoside, isoacteoside and 2'-acetylacteoside significantly restored ern blotting. All data was shown as the mean $\pm \mathrm{SD}$. \# $=p<0.05$, $\# \#=p<0.01$, \#\#\#=p<0.001, compared with non-irradiated group. $*=p<0.05, * *=p<0.01, * * *=p<0.001$ compared with only UVBirradiated group

UVB-induced down-regulation of type I procollagen. Meanwhile, the impaired TGF- $\beta /$ Smad signaling pathway also was reversed. Among them, acteoside showed the best effect on p-Smad2/3 and Smad7 protein, and 2'-acetylacteoside improved the TGF- $\beta$ expression greatly.TARC is a chemokine which plays a key role in Th2-mediated inflammatory diseases [37]. Hino et al. [14] reported that exposing $\mathrm{HaCaTs}$ to narrow UVB radiation inhibited TNF- $\alpha /$ IFN- $\gamma$-stimulated TARC expression and the expression of TARC was turned off in UVB-irradiated or non-irradiated HaCaTs. However, in our study, weak basal expression of TARC was detected in normal HaCaTs. NF- $\kappa \beta$ was known to play a key role in induction of TARC chemokine [38]. In this study, UVB radiation was found to significantly stimulate the activation of NF- $\kappa \beta$, which might be the main cause of TARC up-regulation. Recently, Jang et al. [17] reported that UVB could stimulate TSLP expression by activation of MAPK signaling pathway. They suggested that the up-regulation of TSLP might increase TARC production by activation of dendritic cells. These result suggested that MAPK might be involved in TARC expression. Therefore, the effects of MAPK inhibitors on TARC expression in UVBirradiated $\mathrm{HaCaTs}$ were further examined. The JNK inhibitor, SP600125, was found to inhibit UVB-induced TARC expression, while ERK inhibitor, PD98059 appeared no 

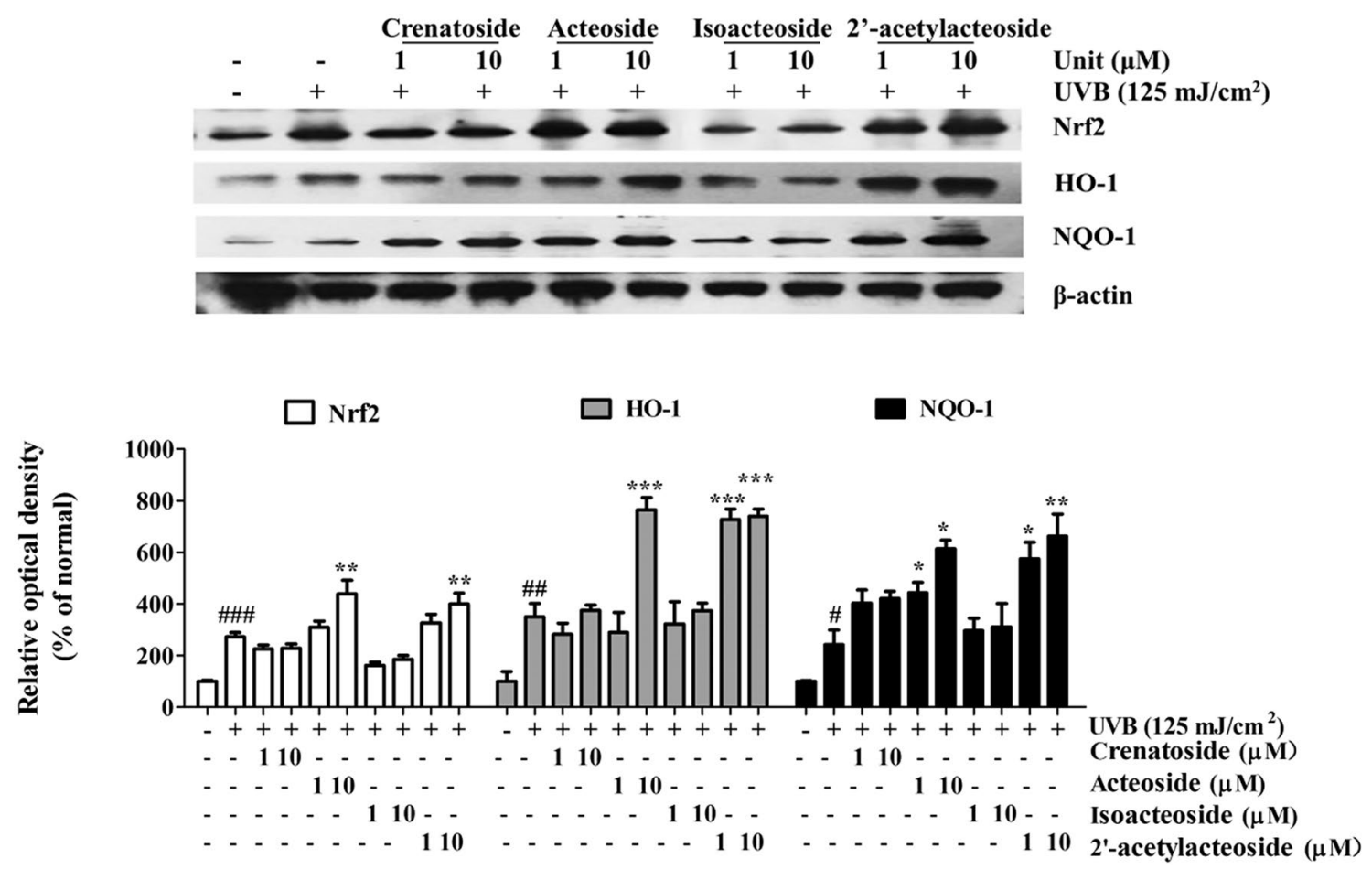

Fig. 13 Effects of crenatoside, acteoside, isoacteoside and 2'-acetylacteoside on Nrf2 pathway. After UVB irradiation, cells were treated with different concentrations of samples for $3 \mathrm{~h}$. The levels of Nrf2, HO-1 and NQO-1 were detected by western blotting. All data was

effects on TARC production in HaCaT cells. These results indicated that JNK but not ERK might be involved in the activation of NF- $\kappa \beta$ that was required for the induction of TARC. Thus, we further examined whether treatment of HaCaTs with PhGs affected UVB-stimulated TARC expression. As expected, acteoside and 2'-acetylacteoside showed a down-regulation on the TARC expression, which might be involved in blocking activation of NF- $\kappa \beta$ and JNK phosphorylation. The TARC is believed to play an important role in the pathogenesis of skin diseases, such as atopic dermatitis, bullous pemphigoid, mycosis fungoides and systemic lupus erythematosus [39, 40]. Therefore, acteoside and 2'-acetylacteoside might be candidate materials for treating TARCrelated skin diseases. However, the biological effects of UVB-induced TSLP and TARC on photo-damage still need to be further explored.

It is well known that UV-activated protein kinase cascades were mediated by ROS. UV-stimulated ROS not only induces the progressive deterioration of cellular structure and function, but also results in chemical modifications to cell macromolecules like DNA, proteins and fatty acids. The cell protection function depends on elaborate antioxidant defense system of enzymatic and non-enzymatic pathway. Nrf2 is essential for the antioxidant responsive element (ARE)-mediated antioxidants, such as HO-1 and shown as the mean $\pm \mathrm{SD} . \#=p<0.05, \# \#=p<0.01, \# \# \#=p<0.001$, compared with non-irradiated group. $*=p<0.05$, ** $=p<0.01$, $* * *=p<0.001$ compared with only UVB-irradiated group

NQO-1 expression [41]. Seo et al. [42] showed that acteoside could stimulate HO- 1 expression through activation of Nrf2 in LPS-induced RAW 264.7 cells. Besides, Wang et al. [43] reported that acteoside protected PC12 cells against $\mathrm{A} \beta$-induced oxidative damage by activation of $\mathrm{Nrf} 2$ nuclear translocation and HO-1 antioxidant expression. Whether the inhibitory effects of PhGs on ROS are regulated by activation of Nrf2 pathway are unknown, so the effects of PhGs on Nrf2 pathway were investigated. The results indicated that acteoside and 2'-acetylacteoside effectively stimulated Nrf2 expression. Meanwhile, HO-1 and NQO-1 were also significantly up-regulated, suggesting that they protected HaCaTs from UVB-induced oxidative damage by activating endogenous antioxidant pathway (Fig. 13).

Generally speaking, excessive exposure to UV radiation causes ROS formation, inflammation and collagen degradation, which are the main reasons for skin photodamage. The process of skin photo-aging is complex and can be triggered by various biological pathways, including receptor-initiated signaling, oxidative stress, telomerebased DNA damage, mitochondrial damage and apoptosis. It appears that acteoside and 2'-acetylacteoside could effectively inhibit UVB-induced photo-damage by downregulation of TARC and ROS production, as well as collagen degradation. The photoprotective mechanism was 

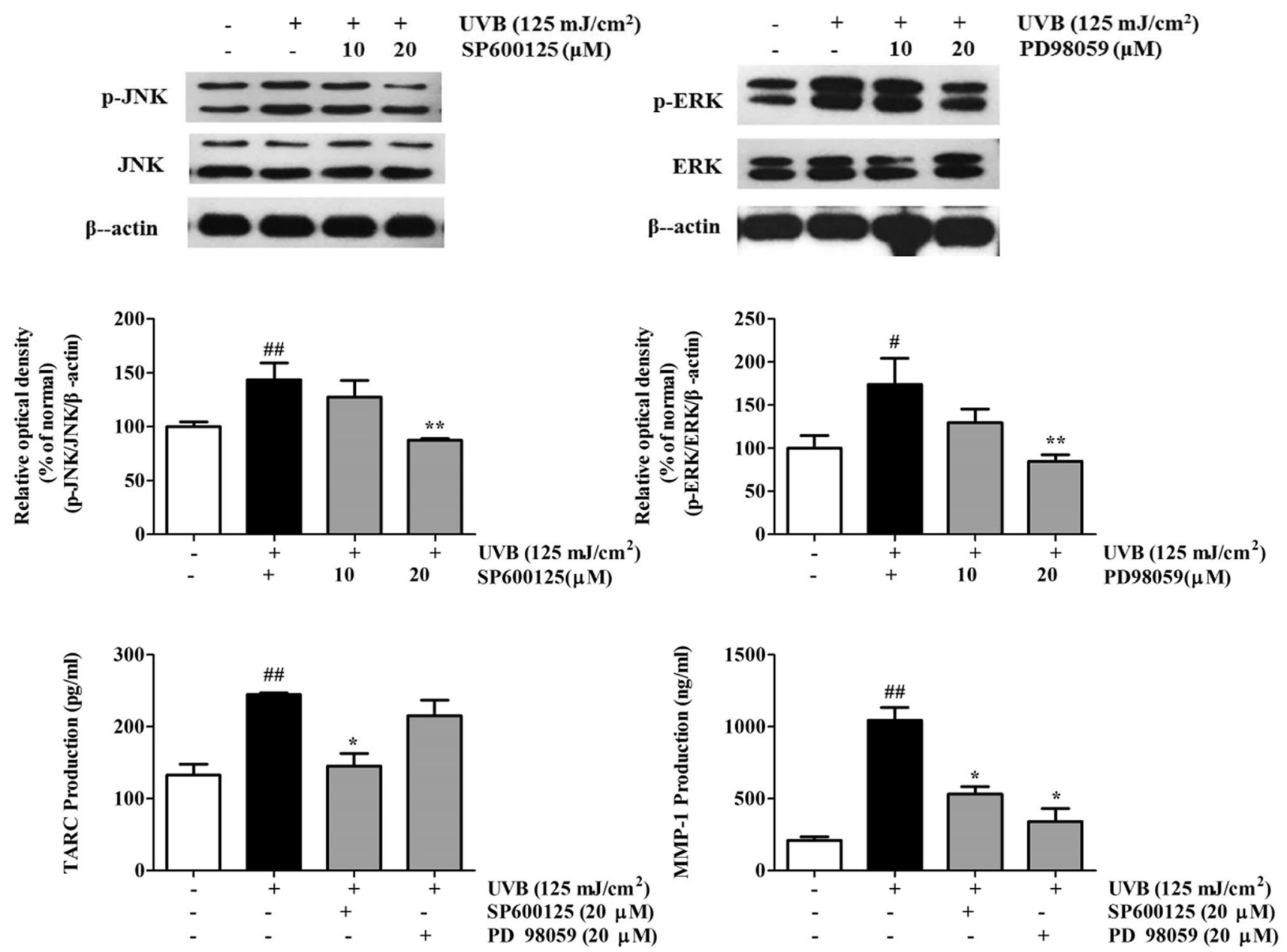

Fig. 14 Effects of MAPK inhibitors on UVB-inducd MMP-1 and TARC production. HaCaT cells were pre-treated with indicated concentrations of inhibitors (PD98590 and SP600125) for $2 \mathrm{~h}$, and then exposed with UVB radiation. After incubation of $1 \mathrm{~h}$, the protein levels of JNK, ERK and their phosphorylation form were measured by

involved in inhibition of MAPK/AP- 1, I $\kappa-\beta \alpha / \mathrm{NF}-\kappa \beta$, and activation of Nrf2, TGF- $\beta /$ Smad pathway. Acteoside and 2 '-acetylacteoside might be served as good candidates in cosmetics and functional foods industry, as well as new drug research and development. However, further study should be performed to elucidate the effect of PhGs and the underlying mechanisms in vivo and clinical trials.

Acknowledgements This work was supported by the TAEYI Life Science Co. Ltd., the Natural Science Foundation of Anhui Province (2008085QH417), Bengbu city and Bengbu Medical College jointed science and technology key projects (BYLK201823), the Natural Science Research Project of Anhui Educational Committee (KJ2020A0565), and the Key Technologies R \& D Program of Bengbu Medical College (BYKY1807ZD). western blotting. After incubation of $72 \mathrm{~h}$, the secreted MMP-1 and TARC were determined by ELISA kits. The result was shown as the mean $\pm \mathrm{SD}$. $\#=p<0.05, \# \#=p<0.01$, compared with non-irradiated group. ${ }^{*}=p<0.05, * *=p<0.01$, compared with only UVB-irradiated group

\section{Declarations}

Conflict of interest There are no conflicts to declare.

\section{References}

1. Zheng, H., Zhang, M., Luo, H., \& Li, H. (2019). Isoorientin alleviates UVB-induced skin injury by regulating mitochondrial ROS and cellular autophagy. Biochemical and Biophysical Research Communications, 514, 1133-1139.

2. Kammeyer, A., \& Luiten, R. M. (2015). Oxidation events and skin aging. Ageing Research Reviews, 21, 16-29. 
3. Ikehata, H., \& Yamamoto, M. (2018). Roles of the KEAP1-NRF2 system in mammalian skin exposed to UV radiation. Toxicology and Applied Pharmacology, 360, 69-77.

4. Blackstone, B. N., Wilgus, T. A., Roy, S., Wulff, B. C., \& Powell, H. M. (2020). Skin biomechanics and miRNA expression following chronic UVB irradiation. Advances in wound care, 9, 79-89.

5. Kwon, K. R., Alam, M. B., Park, J. H., Kim, T. H., \& Lee, S. H. (2019). Attenuation of UVB-induced photo-aging by polyphenolic-rich spatholobus suberectus stem extract via modulation of MAPK/AP-1/MMPs signaling in human keratinocytes. Nutrients, $11,1-14$.

6. Xuan, S. H., Park, Y. M., Ha, J. H., Jeong, Y. J., \& Park, S. N. (2017). The effect of dehydroglyasperin C on UVB-mediated MMPs expression in human HaCaT cells. Pharmacological Reports: PR, 69, 1224-1231.

7. Gao, W., Wang, Y. S., Hwang, E., Lin, P., Bae, J., Seo, S. A., Yan, Z., \& Yi, T. H. (2018). Rubus idaeus L. (red raspberry) blocks UVB-induced MMP production and promotes type I procollagen synthesis via inhibition of MAPK/AP-1, NF-kappabeta and stimulation of TGF-beta/Smad, Nrf2 in normal human dermal fibroblasts. Journal of Photochemistry and Photobiology B, Biology, 185, 241-253.

8. Song, H., Zhou, Y., Peng, A., Liu, J., Wu, X., Chen, W., \& Liu, Z. (2020). Aurora-B promotes osteosarcoma cell growth and metastasis through activation of the NPM1/ERK/NF-kappabeta/MMPs Axis. Cancer Management and Research, 12, 4817-4827.

9. Mohamed, M. A., Jung, M., Lee, S. M., Lee, T. H., \& Kim, J. (2014). Protective effect of Disporum sessile D.Don extract against UVB-induced photoaging via suppressing MMP-1 expression and collagen degradation in human skin cells. Journal of Photochemistry and Photobiology B, Biology, 133, 73-79.

10. Ali, F., Khan, B. A., \& Sultana, S. (2016). Wedelolactone mitigates UVB induced oxidative stress, inflammation and early tumor promotion events in murine skin: Plausible role of NFkB pathway. European Journal of Pharmacology, 786, 253-264.

11. Matthew, P. V., \& Constance, E. B. (2002). Transcriptional regulation of collagenase (MMP-1, MMP-13) genes in arthritis: Integration of complex signaling pathways for the recruitment of genespecific transcription factors. Arthritis Research, 4, 157-164.

12. Yu, B., Koga, T., Urabe, K., Moroi, Y., Maeda, S., Yanagihara, Y., \& Furue, M. (2002). Differential regulation of thymus-and activation-regulated chemokine induced by IL-4, IL-13, TNF- $\alpha$ and IFN- $\gamma$ in human keratinocyte and fibroblast. Journal of Dermatological Science, 30, 29-36.

13. Park, J. H., Kim, M. S., Jeong, G. S., \& Yoon, J. (2015). Xanthii fructus extract inhibits TNF-alpha/IFN-gamma-induced Th2chemokines production via blockade of NF-kappaB, STAT1 and p38-MAPK activation in human epidermal keratinocytes. Journal of Ethnopharmacology, 171, 85-93.

14. Hino, R., Kobayashi, M., Mori, T., Orimo, H., Shimauchi, T., Kabashima, K., \& Tokura, Y. (2007). Inhibition of T helper 2 chemokine production by narrowband ultraviolet B in cultured keratinocytes. British Journal of Dermatology, 156, 830-837.

15. Zheng, X. Y., Nakamura, K., Michiko, T., Akiba, H., Oyama, N., NishibuI, A., Kaneko, F., Tsuneml, Y., Kakinuma, T., \& Saeki, H. (2003). Ultraviolet A irradiation inhibits thymus-and activationregulated chemokine (TARC/CCL17) production by a human keratinocyte HaCaT cell line. European Journal of Dermatology, 13, 348-353.

16. Rutt, V. L., Reed, K. X., Liu, X., Richard, E. G., \& Purcell, S. M. (2017). Photosensitive atopic dermatitis exacerbated by UVB exposure. Cutis, 100, 180-184.

17. Jang, Y., Jeong, S. H., Park, Y. H., Bae, H. C., Lee, H., Ryu, W. I., Park, G. H., \& Son, S. W. (2013). UVB induces HIF-1 $\alpha-$ dependent TSLP expression via the JNK and ERK pathways. The Journal of Investigative Dermatology, 133, 2601-2608.
18. Soumelis, V., Reche, P. A., Kanzler, H., Yuan, W., Edward, G., Homey, B., Gilliet, M., Ho, S., Antonenko, S., \& Lauerma, A. (2002). Human epithelial cells trigger dendritic cell-mediated allergic inflammation by producing TSLP. Nature Immunology, $3,673-680$.

19. Liu, S., You, L., Zhao, Y., \& Chang, X. (2018). Hawthorn polyphenol extract inhibits UVB-induced skin photoaging by regulating MMP expression and type I procollagen production in mice. Journal of Agriculture and Food Chemistry, 66, 8537-8546.

20. Kim, C. R., Kim, Y. M., Lee, M. K., Kim, I. H., Choi, Y. H., \& Nam, T. J. (2017). Pyropia yezoensis peptide promotes collagen synthesis by activating the TGF-beta/Smad signaling pathway in the human dermal fibroblast cell line Hs27. International Journal of Molecular Medicine, 39, 31-38.

21. Verrecchia, F., Chu, M. L., \& Mauviel, A. (2001). Identification of novel TGF- $\beta /$ Smad gene targets in dermal fibroblasts using a combined cDNA microarray/promoter transactivation approach. Journal of Biological Chemistry, 276, 17058-17062.

22. Gao, W., Lin, P., Hwang, E., Wang, Y., Yan, Z., Ngo, H. T. T., \& Yi, T. H. (2018). Pterocarpus santalinus L. regulated ultraviolet $\mathrm{b}$ irradiation-induced procollagen reduction and matrix metalloproteinases expression through activation of tgf-beta/smad and inhibition of the MAPK/AP-1 pathway in normal human dermal fibroblasts. Photochemistry and Photobiology, 94, 139-149.

23. Qu, Z. Y., Zhang, Y. W., Yao, C. L., Jin, Y. P., Zheng, P. H., Sun, C. H., Liu, J. X., Wang, Y. S., \& Wang, Y. P. (2015). Chemical constituents from Orobanche cernua Loefling. Biochemical Systematics and Ecology, 60, 199-203.

24. Gao, W., Wang, Y. S., Qu, Z. Y., Hwang, E., Ngo, H. T. T., Wang, Y. P., Bae, J., \& Yi, T. H. (2018). Orobanche cernua loefling attenuates ultraviolet B-mediated photoaging in human dermal fibroblasts. Photochemistry and Photobiology, 94, 733-743.

25. Hwang, E., Park, S. Y., Sun, Z. W., Shin, H. S., Lee, D. G., \& Yi, T. H. (2013). The protective effects of fucosterol against skin damage in UVB-irradiated human dermal fibroblasts. Marine Biotechnology, 16, 361-370.

26. Parker, C. (2009). Observations on the current status of Orobanche and Striga problems worldwide. Pest Management Science, 65, 453-459.

27. Kostyuk, V., Potapovich, A., Lulli, D., Stancato, A., De-Luca, C., Pastore, S., \& Korkina, L. (2013). Modulation of human keratinocyte responses to solar UV by plant polyphenols as a basis for chemoprevention of non-melanoma skin cancers. Current Medicinal Chemistry, 20, 869-879.

28. Mazzon, E., Esposito, E., Di Paola, R., Riccardi, L., Caminiti, R., Dal Toso, R., Pressi, G., \& Cuzzocrea, S. (2009). Effects of verbascoside biotechnologically produced by Syringa vulgaris plant cell cultures in a rodent model of colitis. Naunyn-Schmiedeberg's Archives of Pharmacology, 380, 79-94.

29. Espinosa-González, A. M., García-Bores, A. M., Benítez-Flores, J. D. C., Sandoval-Pérez, C. E., González-Valle, M. D. R., Céspedes, C. L., \& Avila-Acevedo, J. G. (2016). Photoprotective effect of verbascoside from Buddleja cordata in SKH-1 mice exposed to acute and chronic UV-B radiation. Boletin Latinoamericano y del Caribe de Plantas Medicinales y Aromaticas, 15, 288-300.

30. Chae, S., Kim, J. S., Kang, K. A., Bu, H. D., Lee, Y., Seo, Y. R., Hyun, J. W., \& Kang, S. S. (2005). Antioxidant activity of isoacteoside from Clerodendron trichotomum. Journal of Toxicology \& Environmental Health Part A: Current Issues, 68, 389-400.

31. Nam, S. Y., Kim, H. Y., Yoou, M. S., Kim, A. H., Park, B. J., Jeong, H. J., \& Kim, H. M. (2015). Anti-inflammatory effects of isoacteoside from Abeliophyllum distichum. Immunopharmacology and Immunotoxicology, 37, 258-264.

32. Shen, T., Li, X., Hu, W., Zhang, L., Xu, X., Wu, H., \& Ji, L. (2015). Hepatoprotective effect of phenylethanoid glycosides from 
Incarvillea compacta against $\mathrm{CCl}_{4}$-induced cytotoxicity in $\mathrm{HepG} 2$ cells. J Korean Soc Appl Biol Chem, 58, 617-625.

33. Yu, Q., Li, X., \& Cao, X. (2016). Cardioprotective effects of phenylethanoid glycoside-rich extract from Cistanche deserticola in ischemia-reperfusion-induced myocardial infarction in rats. Annals of Vascular Surgery, 34, 234-242.

34. Van Doren, S. R. (2015). Matrix metalloproteinase interactions with collagen and elastin. Matrix Biology: Journal of the International Society for Matrix Biology, 44-46, 224-231.

35. Shin, M. K., Lee, J. W., Kim, Y. I., Kim, Y. O., Seok, H., \& Kim, N. I. (2014). The effects of platelet-rich clot releasate on the expression of MMP-1 and type I collagen in human adult dermal fibroblasts: PRP is a stronger MMP-1 stimulator. Molecular biology reports, 41, 3-8.

36. Quan, T., He, T., Voorhees, J. J., \& Fisher, G. J. (2001). Ultraviolet irradiation blocks cellular responses to transforming growth factor- $\beta$ by down-regulating its type-II receptor and inducing Smad7. Journal of Biological Chemistry, 276, 26349-26356.

37. Nguyen, C. T. H., Kambe, N., Ueda-Hayakawa, I., Kishimoto, I., Ly, N. T. M., Mizuno, K., \& Okamoto, H. (2018). TARC expression in the circulation and cutaneous granulomas correlates with disease severity and indicates Th2-mediated progression in patients with sarcoidosis. Allergology International: Official Journal of the Japanese Society of Allergology, 67, 487-495.

38. Ahn, S., Siddiqi, M. H., Aceituno, V. C., Simu, S. Y., Zhang, J., Jimenez Perez, Z. E., Kim, Y. J., \& Yang, D. C. (2016).
Ginsenoside Rg5:Rk1 attenuates TNF-alpha/IFN-gamma-induced production of thymus- and activation-regulated chemokine (TARC/CCL17) and LPS-induced NO production via downregulation of NF-kappaB/p38 MAPK/STAT1 signaling in human keratinocytes and macrophages, In vitro cellular \& developmental biology. Animal, 52, 287-295.

39. Saeki, H., \& Tamaki, K. (2006). Thymus and activation regulated chemokine (TARC)/CCL17 and skin diseases. Journal of Dermatological Science, 43, 75-84.

40. Okamoto, H., Koizumi, K., Yamanaka, H., Saito, T., \& Kamatani, N. (2003). A role for TARC/CCL17, a CC chemokine, in systemic lupus erythematosus. Journal of Rheumatology, 30, 2369-2373.

41. Marrot, L., Jones, C., Perez, P., \& Meunier, J. R. (2008). The significance of Nrf2 pathway in (photo)-oxidative stress response in melanocytes and keratinocytes of the human epidermis. Pigment Cell \& Melanoma Research, 21, 79-88.

42. Seo, E. S., Oh, B. K., Pak, J. H., Yim, S. H., Gurunathan, S., Kim, Y. P., \& Lee, K. J. (2013). Acteoside improves survival in cecal ligation and puncture-induced septic mice via blocking of high mobility group box 1 release. Molecules and Cells, 35, 348-354.

43. Wang, H. Q., Xu, Y. X., \& Zhu, C. Q. (2012). Upregulation of heme oxygenase- 1 by acteoside through ERK and PI3-K/Akt pathway confer neuroprotection against beta-amyloid-induced neurotoxicity. Neurotoxicity Research, 21, 368-378. 